Received 00th January 20xx, Accepted 00th January 20xx DOI: $10.1039 / x 0 \times x 00000 x$

\title{
Synthesis, crystal structure, and optical properties of fluorinated poly(pyrazole) ligands and in-silico assessment of their affinity for volatile organic compounds ${ }^{\dagger}$
}

\author{
Alessandro Pedrini, ${ }^{a}$ Angelo Maspero, ${ }^{\mathrm{b},{ }^{*}}$ Silvia Bracco, ${ }^{a}$ Angelina Comotti, ${ }^{a}$ Simona Galli, ${ }^{b}$ Luciano Marchiò, ${ }^{\mathrm{c}}$ Luca Nardo, ${ }^{\mathrm{b}}$
}

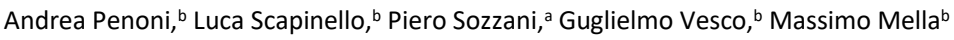

\begin{abstract}
Three new fluorinated bis(pyrazoles), namely: 1,4-bis(1 $\mathrm{H}$-pyrazol-4-ylethynyl)-2-fluorobenzene ( $\left.\mathrm{H}_{2} \mathrm{BPEFB}\right), 1,4-$ bis$(1 \mathrm{H}$ pyrazol-4-ylethynyl)-2,3-difluorobenzene $\left(\mathrm{H}_{2} \mathrm{BPEF}_{2} \mathrm{~B}\right)$ and 1,4-bis(1H-pyrazol-4-ylethynyl)-tetrafluorobenzene $\left(\mathrm{H}_{2} \mathrm{BPEF}{ }_{4} \mathbf{B}\right)$ have been synthesized taking advantage of Sonogashira coupling reactions, and characterized as per their crystal and molecular structure, spectroscopic and dielectric properties, and hydrophobicity. In the crystal structures, the three molecules, whose deviation from planarity increases at increasing the fluorination degree, interact by means of hydrogen bonds, forming 2D supramolecular layers. Notably, the absorption and fluorescence emission properties are only slightly affected by the fluorination degree in both the solid state and solution. Furthermore, the spectral line-shapes are weakly dependent on the environment when dissolved in a number of solvents of different polarity and hydrogen-bonding affinity. On the other hand, the dielectric constant monotonically increases at increasing the number of fluorine atoms. In silico molecular modeling with the time-resolved density functional theory has offered a valuable mean to rationalize the above mentioned behaviors and has shed some light on the ligands affinity towards representative gases $-\mathrm{H}_{2} \mathrm{O}$ and $\mathrm{CO}_{2}$ - and organic solvents - toluene.
\end{abstract}

\section{Introduction}

Pyrazole and its derivatives have been extensively studied for their wide biological activities, ${ }^{1-3}$ including antibacterial, ${ }^{4}$ antifungal, ${ }^{5-8}$ and insecticidal ${ }^{9-11}$ properties. Furthermore, pyrazoles have been intensively employed as nitrogen-donor ligands in coordination chemistry. ${ }^{12}$ After deprotonation, pyrazolates may show up to twenty different terminal or bridging coordination modes, ${ }^{13}$ due to the two donor atoms they possess. As part of di- or poly-topic ligands in coordination compounds, these heterocyclic rings have found a plethora of applications in different fields, ranging from catalysis ${ }^{14-16}$ to medicine ${ }^{17}$ In the past two decades, poly(pyrazole)-based ligands

\footnotetext{
a. University of Milano Bicocca, Dept. of Materials Sciences, Via Cozzi, 55 - 20137 Milano (MI), Italy.

b. University of Insubria, Dept. of Science and High Technology, Via Valleggio, 11 22100 Como (CO), Italy.

University of Parma, Dept. of Dipartimento di Scienze Chimiche, della Vita e della Sostenibilità Ambientale, Parco Area delle Scienze 17/A - 43100 Parma, Italy.

+ Electronic Supplementary Information (ESI) available: ATR-FTIR spectra and simultaneous thermal analyses of $\mathbf{H}_{2}$ BPEFB, $\mathbf{H}_{2} \mathbf{B P E F}_{2} \mathbf{B}$ and $\mathbf{H}_{2} \mathrm{BPEF}_{4} \mathbf{B}$ (Figs. S1-S6). ${ }^{1} \mathrm{H},{ }^{13} \mathrm{C}$ and ${ }^{19} \mathrm{~F}$ NMR spectra of intermediates 2a-2c (Figs. S7-S15). ${ }^{1} \mathrm{H},{ }^{13} \mathrm{C}$ and ${ }^{19} \mathrm{~F}$ NMR spectra of $\mathbf{H}_{2}$ BPEFB, $\mathbf{H}_{2} \mathbf{B P E F}_{2} \mathbf{B}$ and $\mathbf{H}_{2} \mathrm{BPEF}_{4} \mathrm{~B}$ (Figs. S16-S24). Crystal data and structure refinement details for $\mathrm{H}_{2} \mathrm{BPEFB}, \mathrm{H}_{2} \mathrm{BPEF}_{2} \mathrm{~B}$ and $\mathrm{H}_{2} \mathrm{BPEF}_{4} \mathrm{~B}$ (Table S1). Representation of the molecular structures of $\mathrm{H}_{2} B$ PEFB, $\mathrm{H}_{2} \mathrm{BPEF}_{2} \mathrm{~B}$ and $\mathrm{H}_{2} \mathrm{BPEF}_{4} \mathrm{~B}$ (Figs. S25-S27). Representation of the crystal structures of $\mathbf{H}_{2} \mathbf{B P E F B}$ and $\mathbf{H}_{2} \mathbf{B P E F}_{4} \mathbf{B}$ (Figs. S28, S29). Solid-state absorption and fluorescence spectral line-shapes of $\mathrm{H}_{2}$ BPEFB, $\mathrm{H}_{2} \mathrm{BPEF}_{2} \mathrm{~B}$ and $\mathrm{H}_{2} \mathrm{BPEF}_{4} \mathrm{~B}$ (Fig. S30). Absorption spectra of $\mathrm{H}_{2}$ BPEFB $\mathrm{H}_{2} \mathrm{BPEF}_{2} \mathrm{~B}$ and $\mathrm{H}_{2} \mathrm{BPEF}_{4} \mathrm{~B}$ in different solvents (Fig. S31). Fluorescence emission spectra of $\mathrm{H}_{2} \mathrm{BPEFB}, \mathrm{H}_{2} \mathrm{BPEF}_{2} \mathrm{~B}$ and $\mathrm{H}_{2} \mathrm{BPEF}_{4} \mathrm{~B}$ in different solvents (Fig. S32). Powder X-ray diffraction patterns of $\mathrm{H}_{2} \mathrm{BPEFB}, \mathrm{H}_{2} \mathrm{BPEF}_{2} \mathrm{~B}$ and $\mathrm{H}_{2} \mathrm{BPEF}_{4} \mathrm{~B}$ before and after pellettization (Fig. S33). Behavior of $\kappa$ for $\mathrm{H}_{2} B$ PEFB and $\mathbf{H}_{2} \mathrm{BPEF}_{2} \mathrm{~B}$ after exposure to water vapour (Fig. S34). See DOI: 10.1039/x0xx00000x.
}

have been deeply explored as building blocks for metal-organic frameworks (MOFs) and coordination polymers (CPs). ${ }^{18}$ The search for CPs and MOFs with industrially and technologically relevant functional properties has prompted the thriving engineering and synthesis of new poly(pyrazoles). ${ }^{19}$ Due to their intrinsic porosity, MOFs are ideal candidates for, e.g., adsorption and separation, $20-23$ heterogeneous catalysis ${ }^{24-30}$ as well as sensing. ${ }^{31-36}$ Together with modifying the nature and length of its skeleton, functionalizing the ligand was found to be a powerful tool for the modulation of MOFs properties. ${ }^{37-42}$ In the past ten years, particular attention was dedicated to fluorine-functionalized poly(azoles) and the corresponding MOFs. ${ }^{43-49}$ Replacement of hydrogen with fluorine profoundly changes the chemico-physical properties. C-F bonds are stronger than $\mathrm{C}-\mathrm{H}$ bonds; moreover, the dipolar moment of the $\mathrm{C}-\mathrm{F}$ moiety is higher and has opposite direction as compared to that of the $\mathrm{C}-\mathrm{H}$ bond. Finally, the high electronegativity of fluorine makes the $\mathrm{C}-\mathrm{F}$ group less polarizable than the $\mathrm{C}-\mathrm{H}$ group.

Fluorine substitution may strongly affect the photophysical and photoluminescence properties of hetero-aromatic compounds, potentially resulting in either enhancement or dramatic reduction of the luminescence quantum yields. ${ }^{50,51}$ On the other hand, fluorescence preservation upon fluorination is a very promising prerequisite to devise optical sensors for volatile organic compounds (VOCs), in case $[\pi-\pi]$ stacking interactions are established between the perfluorinated ligands and (VOCs) themselves. Indeed, $[\pi-\pi]$ stacking is known to result in massive fluorescence depression. Due to the increased chemical stability conferred by fluorination, ${ }^{43}$ fluorescent MOFs capable of sequestering VOCs from an aqueous 
environment and sense their presence through VOCs-concentration dependent fluorescence quenching might even be envisaged.

Recently, ${ }^{52,53}$ we reported the synthesis and characterization of the poly-pyrazole ligand 1,4-bis( $1 \mathrm{H}$-pyrazol-4-ylethynyl)benzene, $\mathbf{H}_{2}$ BPEB (Scheme 1) and showed that it is capable to form thermally stable MOFs with $3 d$ transition metals [Fe(III), Ni(II) and $\mathrm{Zn}(\mathrm{II})]$. The presence, in the skeleton of this ligand, of two ethynyl groups promotes the fluorescence of the compound by ensuring full conjugation throughout the structure. In this article we report the synthesis of three new fluorine-functionalized pyrazole ligands derived from $\mathbf{H}_{2}$ BPEB, namely: 1,4-bis( $1 \mathrm{H}$-pyrazol-4-ylethynyl)-2fluorobenzene ( $\left.\mathrm{H}_{2} \mathrm{BPEFB}\right), \quad$ 1,4-bis(1H-pyrazol-4-ylethynyl)-2,3difluorobenzene $\left(\mathbf{H}_{2} \mathbf{B P E F}_{2} \mathbf{B}\right)$ and 1,4-bis $(1 \mathrm{H}$-pyrazol-4-ylethynyl)tetrafluorobenzene $\left(\mathrm{H}_{2} \mathbf{B P E F}_{4} \mathbf{B}\right)$ (Scheme 1). After determining their crystal structure, we have undertaken an in-depth spectroscopic characterization, demonstrating that they conserve the high fluorescence quantum yield of $\mathrm{H}_{2} \mathrm{BPEB}$, and in-silico calculations, suggesting that fluorination dramatically increases the ligand affinity for the model VOC toluene. These findings are propaedeutic to the future synthesis of a family of highly fluorescent MOFs endowed with high affinity for VOCs.

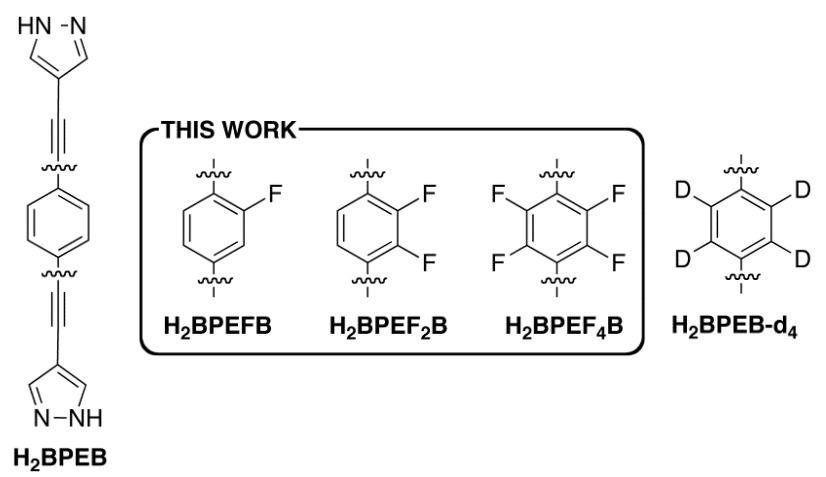

Scheme 1 Molecular structures of 1,4-bis(1H-pyrazol-4ylethynyl)benzene $\left(\mathrm{H}_{2} \mathrm{BPEB}\right)$ and its known derivatives - the three fluorinated bis(pyrazolyl) ligands reported in this work are evidenced.

\section{Experimental}

\subsection{Materials and methods}

All chemicals and solvents were purchased from commercial sources and used without further purification. Literature procedures were applied in order to synthesize the starting materials 1,4-diethynyl-2fluorobenzene ${ }^{54}$ and 4-iodo-(1-ethoxyethyl)pyrazole, 55 while the compounds 1,4-diethynyl-2,3-difluorobenzene and 1,4-diethynyltetrafluorobenzene were obtained by slight modifications of the procedure used for 1,4-diethynyl-2-fluorobenzene.

${ }^{1} \mathrm{H},{ }^{13} \mathrm{C}(\mathrm{APT})$ and ${ }^{19} \mathrm{~F}$ solution NMR spectra were recorded at 400 , 100 and $376 \mathrm{MHz}$, respectively, on a Bruker Avance 400 spectrometer. ${ }^{1} \mathrm{H}$ and ${ }^{13} \mathrm{C}$ NMR data are reported as follows: chemical shifts (in ppm and referenced to internal TMS), multiplicity ( $s=$ singlet, $b s=$ broad singlet, $d=$ doublet, $d d=$ doublet of doublets, $d m$ $=$ doublet of multiplets, $\mathrm{t}=$ triplet, $\mathrm{q}=$ quartet, $\mathrm{m}=$ multiplet), integration, coupling constants (in $\mathrm{Hz}$ ) and assignation. ${ }^{19} \mathrm{~F}$ NMR data are reported as follows: chemical shifts (in ppm and referenced to internal $\mathrm{CFCl}_{3}$ ) and multiplicity ( $\mathrm{s}=$ singlet). IR spectra were acquired in attenuated total reflectance (ATR) by means of a Nicolet iS10 instrument over the range $4000-600 \mathrm{~cm}^{-1}$. Bands are classified as sh = sharp, $\mathrm{br}=$ broad, $\mathrm{vs}=$ very strong, $\mathrm{s}=$ strong, $\mathrm{m}=$ medium, and $\mathrm{w}$ = weak. Elemental analyses (\% C, $\mathrm{H}$ and $\mathrm{N})$ were obtained with a Perkin Elmer CHN Analyzer 2400 Series II. Simultaneous thermogravimetric analysis (TGA) and differential scanning calorimetry (DSC) were performed with a Netzsch STA 409 instrument under a flow of $\mathrm{N}_{2}$, in the 303-1173 K temperature range, at a heating rate of $10 \mathrm{~K} / \mathrm{min}$. Powder X-ray diffraction (PXRD) patterns for qualitative analysis prior to the functional characterization were acquired on powdered samples or pressed pellets in the $3-35^{\circ} 2 \theta$ range, with steps of $0.02^{\circ}$ and time/step of 1 $\mathrm{s}$, on a Bruker AXS D8 Advance diffractometer equipped with a $\mathrm{Cu}$ K $\alpha$ tube $(\lambda=1.5418 \AA$ ) , a Bruker Lynxeye linear position-sensitive detector, a filter of nickel in the diffracted beam and the following optics: primary beam Soller slits $\left(2.3^{\circ}\right)$, fixed divergence slit $\left(0.5^{\circ}\right)$, receiving slit $(8 \mathrm{~mm})$. The generator was set at $40 \mathrm{kV}$ and $40 \mathrm{~mA}$.

The solid-state UV-Vis spectra were acquired with a Jasco V-770 spectrophotometer equipped with an integrating sphere module. The UV-Vis spectra in solution were recorded with a Perkin Elmer Lambda2 spectrophotometer on $\sim 1 \mathrm{mM}$ solutions. The solid-state fluorescence spectra were measured by means of a Jasco FP-8500 spectrofluorimeter equipped with a dedicated sample-holder for powdered samples. The powder was submitted to the irradiation beam at $56^{\circ}$ to optimize the signal-to-excitation stray light collection ratio. The fluorescence spectra in solution were recorded with a PTI fluorescence master system spectrofluorimeter on $\sim 1 \mathrm{mM}$ solutions. The instrument was interfaced with the acquisition software Felix 2000 , which performed an online correction of the data with respect to the excitation lamp spectral radiance and detector spectral quantum efficiency.

\subsection{Synthesis of fluorinated 1,4-bis(1H-pyrazol-4-} ylethynyl)benzene ligands

General procedure for the preparation of protected ligands. In a Schlenk tube, the fluorinated diethynylbenzene $(1.54 \mathrm{mmol}$ ) (1a-c in Scheme 2) was dissolved in $40 \mathrm{~mL}$ of a 1:1 mixture of triethylamine (TEA) and tetrahydrofurane (THF). The solution was degassed $(3 \times$ freeze/pump/thaw) and $\mathrm{Pd}\left(\mathrm{PPh}_{3}\right)_{2} \mathrm{Cl}_{2}(3.30 \mathrm{mmol})$ and $\mathrm{Cul}(0.33$ $\mathrm{mmol})$ were added, followed by 4-iodo-(1-ethoxyethyl)pyrazole $(6.24 \mathrm{mmol})$. The reaction mixture was heated at $343 \mathrm{~K}$ for $4 \mathrm{~h}$. After cooling at room temperature, the reaction mixture was filtered over a Celite pad, then washed with ethyl acetate (EtOAc). The solvent was evaporated under reduced pressure affording a brown residue, which was solubilized in EtOAc and washed with $30 \%$ aq. $\mathrm{NH}_{4} \mathrm{OH}$ and water. The organic layer was dried over $\mathrm{Na}_{2} \mathrm{SO}_{4}$ and evaporated under reduced pressure. The crude solid mixture was purified by Soxhlet extraction with pentane. The washings were recovered and followingly purified by flash column chromatography.

1,4-bis(1-ethoxyethylpyrazol-4-ylethynyl)-2-fluorobenzene (2a). Compound 2a (Scheme 2) was obtained as a pale-yellow solid in $27 \%$ yield after flash column chromatography (silica gel, hexane/EtOAc 7:3). Elem. Anal. calc. for $\mathrm{C}_{24} \mathrm{H}_{25} \mathrm{FN}_{4} \mathrm{O}_{2}\left(\mathrm{FW}=420.5 \mathrm{~g} \mathrm{~mol}^{-1}\right)$ : $\mathrm{C}, 68.55$; $\mathrm{H}, 5.99 ; \mathrm{N}, 13.32 \%$; found: C, 68.23; H, 6.20; N, 13.41\%. ${ }^{1} \mathrm{H}$ NMR (400 $\left.\mathrm{MHz}, \mathrm{DMSO}-d_{6}, 298 \mathrm{~K}\right): \delta(\mathrm{ppm}) 8.39(\mathrm{~s}, 1 \mathrm{H}, \mathrm{ArH}), 8.35$ (s, $\left.1 \mathrm{H}, \mathrm{ArH}\right)$, 
$7.81(\mathrm{~s}, 1 \mathrm{H}, \mathrm{ArH}), 7.79(\mathrm{~s}, 1 \mathrm{H}, \mathrm{ArH}), 7.57\left(\mathrm{t}, 1 \mathrm{H}, \mathrm{J}=7.8 \mathrm{~Hz}, \mathrm{ArH}_{6}\right), 7.46$ (dd, $\left.1 \mathrm{H}, \mathrm{J}=10.2 \mathrm{~Hz}, \mathrm{~J}=1.6 \mathrm{~Hz}, \mathrm{ArH}_{5}\right), 7.35(\mathrm{dd}, 1 \mathrm{H}, \mathrm{J}=8.0 \mathrm{~Hz}, \mathrm{~J}=1.6$ $\left.\mathrm{Hz}, \mathrm{ArH}_{3}\right), 5.58(\mathrm{q}, 2 \mathrm{H}, \mathrm{J}=6.0 \mathrm{~Hz}, \mathrm{NCH}), 3.44\left(\mathrm{~m}, 2 \mathrm{H}, \mathrm{OCH}_{2}\right), 3.22(\mathrm{~m}$, $\left.2 \mathrm{H}, \mathrm{OCH}_{2}\right), 1.61\left(\mathrm{~d}, 6 \mathrm{H}, \mathrm{J}=6.0 \mathrm{~Hz}, \mathrm{NCHCH}_{3}\right), 1.05(\mathrm{t}, 6 \mathrm{H}, \mathrm{J}=7.1 \mathrm{~Hz}$, $\mathrm{OCH}_{2} \mathrm{CH}_{3}$ ); ${ }^{13} \mathrm{C}$ NMR (100 MHz, DMSO- $d_{6}, 298 \mathrm{~K}$ ): $\delta$ (ppm) 161.7 (d, $\left.{ }^{1} \mathrm{~J}_{\mathrm{CF}}=248.3 \mathrm{~Hz}\right), 141.8,133.8,132.3,128.0,124.9,118.3\left(\mathrm{~d},{ }^{2} \mathrm{~J}_{\mathrm{CF}}=22.3\right.$ $\mathrm{Hz}), 111.7,102.1,88.8,87.1,85.0,83.3,63.7,21.6,15.2 ;{ }^{19} \mathrm{~F}$ NMR (376 MHz, DMSO- $\left.d_{6}, 298 \mathrm{~K}\right)$ : $\delta(\mathrm{ppm})-110.49(\mathrm{~s}, 1 \mathrm{~F})$; IR (ATR, $\left.\mathrm{cm}^{-1}\right)$ : 3108(w), 3083(w), 2977(w), 2906(w), 2222(s), 1613(w), 1563(w), 1538(w), 1488(s), 1436(m), 1417(m), 1361(m), 1237(w), 1118(vs), 1064(vs), 1009(s), 980(s), 943(s), 864(vs), 826(vs), 787(m), 751(s), 704(s), 663(m), 640(vs).

\section{1,4-bis(1-ethoxyethylpyrazol-4-ylethynyl)-2,3-difluorobenzene}

(2b). Compound $\mathbf{2 b}$ (Scheme 2) was obtained as a pale-yellow solid in $30 \%$ yield after flash column chromatography (silica gel, hexane/EtOAc 8:2). Elem. Anal. calc. for $\mathrm{C}_{24} \mathrm{H}_{24} \mathrm{~F}_{2} \mathrm{~N}_{4} \mathrm{O}_{2}$ (FW $=438.5 \mathrm{~g}$ $\mathrm{mol}^{-1}$ ): C, 65.74; H, 5.52; N, 12.78\%; found: C, 66.01; H, 5.82; N, 12.69\%. ${ }^{1} \mathrm{H}$ NMR (400 MHz, DMSO- $\left.d_{6}, 298 \mathrm{~K}\right): \delta(\mathrm{ppm}) 8.42(\mathrm{~s}, 2 \mathrm{H}$ ArH), $7.84(\mathrm{~s}, 2 \mathrm{H}, \mathrm{ArH}), 7.40(\mathrm{~m}, 2 \mathrm{H}, \mathrm{ArH}), 5.59(\mathrm{q}, 2 \mathrm{H}, \mathrm{J}=6.0 \mathrm{~Hz}, \mathrm{NCH})$, $3.45\left(\mathrm{~m}, 2 \mathrm{H}, \mathrm{OCH}_{2}\right), 3.23\left(\mathrm{~m}, 2 \mathrm{H}, \mathrm{OCH}_{2}\right), 1.61(\mathrm{~d}, 6 \mathrm{H}, \mathrm{J}=6.0 \mathrm{~Hz}$, $\left.\mathrm{NCHCH}_{3}\right), 1.06\left(\mathrm{t}, 6 \mathrm{H}, \mathrm{J}=7.0 \mathrm{~Hz}, \mathrm{OCH}_{2} \mathrm{CH}_{3}\right) ;{ }^{13} \mathrm{C} \mathrm{NMR}(100 \mathrm{MHz}$, DMSO- $\left.d_{6}, 298 \mathrm{~K}\right): \delta(\mathrm{ppm}) 150.2\left(\mathrm{dd},{ }^{1} \mathrm{~J}_{\mathrm{CF}}=252.3 \mathrm{~Hz},{ }^{2} \mathrm{~J}_{\mathrm{CF}}=15.0 \mathrm{~Hz}\right)$, 142.0, 132.6, 128.4, 113.7, 101.7, 90.2, 87.2, 82.3, 63.8, 21.6, 15.2 ${ }^{19} \mathrm{~F}$ NMR (376 MHz, DMSO- $\left.d_{6}, 298 \mathrm{~K}\right) \delta(\mathrm{ppm})-135.80$ (s, 2F); IR (ATR, $\left.\mathrm{cm}^{-1}\right): 3118(\mathrm{w}), 3103(\mathrm{~m}), 2970(\mathrm{~m}), 2916(\mathrm{w}), 2226(\mathrm{~s}), 1610(\mathrm{~m})$, 1569(w), 1531(m), 1484(s), 1429(w), 1410(w), 1360(m), 1233(m), 1110(vs), 1060(vs), 1000(s), 986(m), 940(m), 870(vs), 820(vs), 784(w), 750(s), 710(s), 660(w), 649(vs).

1,4-bis(1-ethoxyethylpyrazol-4-ylethynyl)-tetrafluorobenzene (2c). Compound 2c (Scheme 2) was obtained as a pale-yellow solid in 32\% yield after flash column chromatography (silica gel, hexane/EtOAc 8:2). Elem. Anal. calc. for $\mathrm{C}_{24} \mathrm{H}_{22} \mathrm{~F}_{4} \mathrm{~N}_{4} \mathrm{O}_{2}\left(\mathrm{FW}=474.5 \mathrm{~g} \mathrm{~mol}^{-1}\right)$ : C, 60.76; $\mathrm{H}, 4.67$; N, 11.81\%; found: C, 60.38; H, 4.97; N, 11.77\%. ${ }^{1} \mathrm{H}$ NMR (400 $\left.\mathrm{MHz}, \mathrm{DMSO}-d_{6}, 298 \mathrm{~K}\right): \delta(\mathrm{ppm}) 8.53(\mathrm{~s}, 2 \mathrm{H}, \mathrm{ArH}), 7.91(\mathrm{~s}, 2 \mathrm{H}, \mathrm{ArH})$ $5.60(\mathrm{q}, 2 \mathrm{H}, \mathrm{J}=6.0 \mathrm{~Hz}, \mathrm{NCH}), 3.54\left(\mathrm{~m}, 2 \mathrm{H}, \mathrm{OCH}_{2}\right), 3.23\left(\mathrm{~m}, 2 \mathrm{H}, \mathrm{OCH}_{2}\right)$, $1.61\left(\mathrm{~d}, 6 \mathrm{H}, \mathrm{J}=6.0 \mathrm{~Hz}, \mathrm{NCHCH}_{3}\right), 1.05\left(\mathrm{t}, 6 \mathrm{H}, \mathrm{J}=7.0 \mathrm{~Hz}, \mathrm{OCH}_{2} \mathrm{CH}_{3}\right) ;{ }^{13} \mathrm{C}$ NMR (100 MHz, CDCl $3,298 \mathrm{~K}): \delta(\mathrm{ppm}) 146.4\left(\mathrm{dm},{ }^{1} \mathrm{~J}=249.8 \mathrm{~Hz}\right)$, 142.2, 133.2, 104.4, 101.0, 96.2, 87.3, 75.6, 63.8, 21.5, 15.1; ${ }^{19} \mathrm{~F}$ NMR (376 MHz, DMSO- $\left.d_{6}, 298 \mathrm{~K}\right): \delta(\mathrm{ppm})-138.01(\mathrm{~s}, 4 \mathrm{~F})$; IR (ATR, $\left.\mathrm{cm}^{-1}\right)$ : 3112(w), 3089(w), 2967(w), 2897(w), 2212(s), 1603(w), 1543(w) 1518(w), 1458(s), 1431(m), 1410(m), 1331(m), 1231(w), 1123(vs), 1074(vs), 1001(s), 986(s), 941(s), 869(vs), 827(vs), 787(m), 750(s), 710(s), 661(m), 647(vs).

General procedure for ligands deprotection. The protected ligand $(200 \mathrm{mmol}$ ) (2a-c) was dissolved in $10 \mathrm{~mL}$ of 1,4-dioxane, then $1.5 \mathrm{~mL}$ of $6 \mathrm{M} \mathrm{HCl}$ aqueous solution were added dropwise (Scheme 2). After vigorous stirring at room temperature for 3 hours, the precipitate was filtered, washed with water and dried in vacuo affording the free ligand.

1,4-bis(1H-pyrazol-4-ylethynyl)-2-fluorobenzene ( $\left.\mathrm{H}_{2} \mathrm{BPEFB}\right)$. Offwhite solid, 76\% yield. Elem. Anal. calc. for $\mathrm{C}_{16} \mathrm{H}_{9} \mathrm{FN}_{4}$ (FW $=276.3 \mathrm{~g}$ $\mathrm{mol}^{-1}$ ): C, 69.56; H, 3.28; N, 20.28\%; found: C, 69.47; H, 3.35; N, 20.25\%. ${ }^{1} \mathrm{H}$ NMR (400 MHz, DMSO- $d_{6}, 298 \mathrm{~K}$ ): $\delta$ (ppm) 13.17 (bs, $2 \mathrm{H}$, $\mathrm{NH}), 7.91$ (bs, 4H, ArH), $7.48\left(\mathrm{t}, 1 \mathrm{H}, \mathrm{J}=7.9 \mathrm{~Hz}, \mathrm{ArH}_{6}\right), 7.36$ (dd, $1 \mathrm{H}, \mathrm{J}=$ $\left.10.3 \mathrm{~Hz}, \mathrm{~J}=1.6 \mathrm{~Hz}, \mathrm{ArH}_{5}\right), 7.25\left(\mathrm{dd}, 1 \mathrm{H}, \mathrm{J}=8.0 \mathrm{~Hz}, \mathrm{~J}=1.6 \mathrm{~Hz}, \mathrm{ArH}_{3}\right.$ ); ${ }^{13} \mathrm{C}$ NMR $\left(100 \mathrm{MHz}\right.$, DMSO- $\left.d_{6}, 298 \mathrm{~K}\right): \delta(\mathrm{ppm}) 161.7\left(\mathrm{~d},{ }^{1} \mathrm{~J}_{\mathrm{CF}}=249.2\right.$ $\mathrm{Hz}), 133.7,127.9,125.0,118.2\left(\mathrm{~d},{ }^{2} \mathrm{~J}_{\mathrm{CF}}=22.6 \mathrm{~Hz}\right), 111.7\left(\mathrm{~d},{ }^{2} \mathrm{~J}_{\mathrm{CF}}=16.0\right.$
$\mathrm{Hz}$ ), 100.9, 89.4, 88.7, 85.6, 83.1; ${ }^{19} \mathrm{~F}$ NMR (376 MHz, DMSO- $d_{6}, 298$ $\mathrm{K}): \delta(\mathrm{ppm})-110.64(\mathrm{~s}, 1 \mathrm{~F})$; IR (ATR, $\mathrm{cm}^{-1}$, Fig. S1 of the ESI $\left.{ }^{\dagger}\right): 3095(\mathrm{w})$, 2934(w), 2430(m, br), 2225(m, sh), 1615(w), 1539(m), 1482(m), 1470(m), 1372(m), 1272(w), 1180(w), 1111(m), 1071(w), 994(m), 943(m), 810(s), 742(m). $\mathrm{T}_{\text {dec }}=586 \mathrm{~K}$ (Fig. S2 of the $\left.\mathrm{ESI}^{\dagger}\right)$.

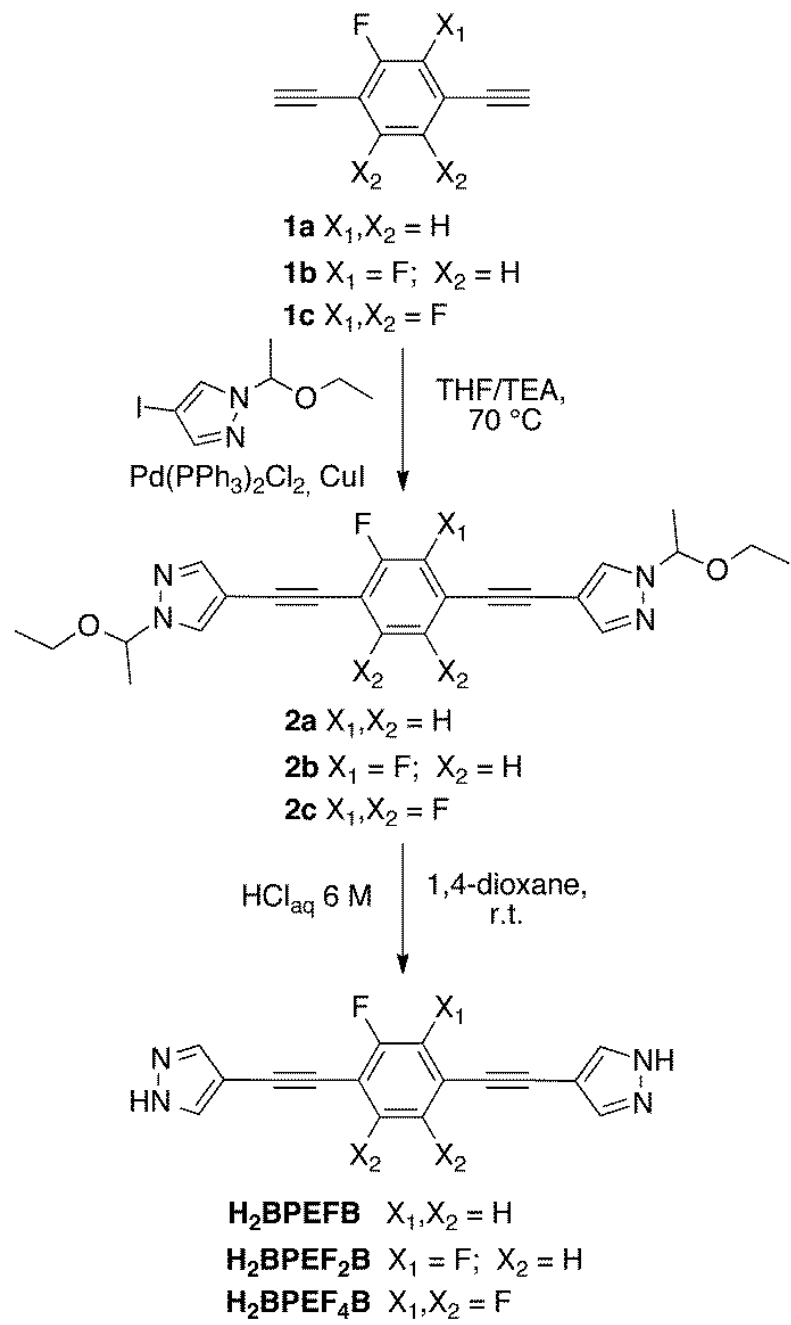

Scheme 2 The two-step synthesis of the mono- $\left(\mathrm{H}_{2} \mathbf{B P E F B}\right)$, di$\left(\mathrm{H}_{2} \mathrm{BPEF}_{2} \mathrm{~B}\right)$ and tetra-fluorinated $\left(\mathrm{H}_{2} \mathrm{BPEF}_{4} \mathrm{~B}\right)$ 1,4-bis $(1 \mathrm{H}$-pyrazol-4ylethynyl)benzene ligands.

1,4-bis(1H-pyrazol-4-ylethynyl)-2,3-difluorobenzene ( $\left.\mathrm{H}_{2} \mathrm{BPEF}_{2} \mathrm{~B}\right)$. Off-white solid, 84\% yield. Elem. Anal. calc. for $\mathrm{C}_{16} \mathrm{H}_{8} \mathrm{~F}_{2} \mathrm{~N}_{4}(\mathrm{FW}=294.3$ $\mathrm{g} \mathrm{mol}^{-1}$ ): C, 65.31; H, 2.74; N, 19.04\%; found: C, 65.12; H, 2.99; N, $18.98 \%{ }^{1} \mathrm{H}$ NMR (400 MHz, DMSO- $d_{6}, 298 \mathrm{~K}$ ): $\delta$ (ppm) 9.82 (bs, $2 \mathrm{H}$, $\mathrm{NH}), 8.03$ (s, 4H, ArH), 7.37 (m, 2H, ArH); ${ }^{13} \mathrm{C}$ NMR (100 MHz, DMSO$\left.d_{6}, 298 \mathrm{~K}\right): \delta(\mathrm{ppm}) 150.1\left(\mathrm{dd},{ }^{1} \mathrm{~J}_{\mathrm{CF}}=252.0 \mathrm{~Hz},{ }^{2} \mathrm{~J}_{\mathrm{CF}}=14.7 \mathrm{~Hz}\right), 137.4$, $128.3,113.7,100.6,90.8,82.1$; ${ }^{19} \mathrm{~F}$ NMR (376 MHz, DMSO- $\left.d_{6}, 298 \mathrm{~K}\right)$ : $\delta(\mathrm{ppm})-135.99$ (s, 2F); IR (ATR, $\mathrm{cm}^{-1}$, Fig. S3 of the ESI $\left.{ }^{\dagger}\right): 3100(\mathrm{w})$, 2924(m), 2426(w), 2220(m), 1610(m), 1534(w), 1492(m), 1475(w), $1378(\mathrm{~m}), 1278(\mathrm{~m}), 1185(\mathrm{~m}), 1121(\mathrm{~m}), 1075(\mathrm{w}), 990(\mathrm{~s}), 948(\mathrm{~s})$, 815(vs), $748(w) . T_{\text {dec }}=576 \mathrm{~K}$ (Fig. S4 of the ESI ${ }^{\dagger}$ ).

1,4-bis(1H-pyrazol-4-ylethynyl)-tetrafluorobenzene $\quad\left(\mathrm{H}_{2} \mathrm{BPEF}_{4} \mathrm{~B}\right)$. Off-white solid, $85 \%$ yield. Elem. Anal. calc. for $\mathrm{C}_{16} \mathrm{H}_{6} \mathrm{~F}_{4} \mathrm{~N}_{4}(\mathrm{FW}=330.2$ $\mathrm{g} \mathrm{mol}^{-1}$ ): C, 58.19; $\mathrm{H}, 1.83 ; \mathrm{N}, 16.97 \%$; found: $\mathrm{C}, 58.10 ; \mathrm{H}, 2.00 ; \mathrm{N}$, 
17.00\%. ${ }^{1} \mathrm{H}$ NMR (400 MHz, DMSO- $\left.d_{6}, 298 \mathrm{~K}\right): \delta(\mathrm{ppm}) 8.10(\mathrm{~s}, 4 \mathrm{H}$, ArH), 5.65 (bs, $2 \mathrm{H}, \mathrm{NH}) ;{ }^{13} \mathrm{C}$ NMR $\left(100 \mathrm{MHz}, \mathrm{CDCl}_{3}, 298 \mathrm{~K}\right): \delta(\mathrm{ppm})$ $146.3\left(\mathrm{dm},{ }^{1} \mathrm{~J}=249.8 \mathrm{~Hz}\right), 137.8,104.4,99.9,96.9,75.5 ;{ }^{19} \mathrm{~F}$ NMR (376 $\left.\mathrm{MHz}, \mathrm{DMSO}-d_{6}, 298 \mathrm{~K}\right): \delta(\mathrm{ppm}):-138.25(\mathrm{~s}, 4 \mathrm{~F})$; IR (ATR, $\mathrm{cm}^{-1}$, Fig. S5 of the $\left.\mathrm{ESI}^{+}\right): 2235(\mathrm{~m}), 1475(\mathrm{~s}), 1379(\mathrm{~m}), 1318(\mathrm{~m}), 1136(\mathrm{w}), 1012(\mathrm{~m})$, 976(s), $939(\mathrm{~m}) . \mathrm{T}_{\mathrm{dec}}=465 \mathrm{~K}$ (Fig. S6 of the $\mathrm{ESI}^{\dagger}$ ).

\subsection{Crystal structure analysis}

Single-crystal $X$-ray diffraction data for $\mathrm{H}_{2} \mathrm{BPEFB}, \mathrm{H}_{2} \mathrm{BPEF}_{2} \mathrm{~B}$ and $\mathrm{H}_{2} \mathrm{BPEF}_{4} \mathrm{~B}$ were collected at $190 \mathrm{~K}$ with a Bruker D8 Photon II area detector diffractometer equipped with a microfocus source (Mo K $\alpha$ : $\lambda=0.71073 \AA$ A). Complete datasets were obtained by merging several series of exposure frames. ${ }^{56}$ The crystals of $\mathrm{H}_{2}$ BPEFB were nonmerohedrally twinned, and two different components were taken into account during data integration and scaling, which was performed with the program TWINABS. ${ }^{57}$ For $\mathbf{H}_{2} \mathrm{BPEF}_{2} \mathrm{~B}$ and $\mathrm{H}_{2} \mathrm{BPEF}_{4} \mathbf{B}$, an absorption correction was applied with the program SADABS. ${ }^{58}$ The crystal structures were solved with Shelx $T^{59}$ and refined on $F^{2}$ with full-matrix least squares with ShelxL, ${ }^{60}$ using the software package Olex2. ${ }^{61}$ Non hydrogen atoms were refined anisotropically, while the hydrogen atoms were placed at their calculated positions and were assigned isotropic thermal parameters. The graphical material was prepared with the program Mercury. ${ }^{62}$ A summary of data collection and structure refinement details for the three ligands is reported in Table S1 of the ESI ${ }^{\dagger}$. CCDC 1968177-1968179 contain the supplementary crystallographic data for this paper.

\subsection{Electronic structure modelling of ligands and ligand-smal molecules interactions}

As for the structural optimization of the isolated ligands, this was carried out at the B3LYP/6-31++g(d,p) level ${ }^{63}$ in the gas phase or employing a continuous solvent model PCM by means of the Gaussian09 suit of codes. ${ }^{64}$ All optimized energies were tested by computing normal modes, which were also useful in interpreting the details of the spectroscopic results. The calculation of the vertical electronic excitations for the energy minimized ligands was conducted at the TD-DFT level using the same Density Function Theory/basis set combination employed for the structural optimization; an identical approach was also exploited to obtain the minimum energy structure for the first excited state of the $\mathrm{H}_{2} \mathrm{BPEFB}$ compound, as well as its normal modes.

When the estimation of intermolecular forces between polar species (i.e. involving electrostatic interactions) was concerned, we selected to use the same theory level used for the study of the isolated ligands. We, instead, opted for the higher MP2/31++g(d,p) level to correctly describe the induced dipole-induced dipole component of the London dispersion forces between the $p$ electronic systems present on both the ligands and toluene molecule. In all cases, Basis Set Superposition Error (BSSE) effects were corrected via the Counterpoise (CP) method. ${ }^{65}$

\subsection{Measurement of the dielectric constant}

The value of the real component of the dielectric constant $(\kappa)$ of samples of $\mathbf{H}_{2} B$ PEFB, $\mathbf{H}_{2} \mathbf{B P E F}_{2} \mathbf{B}$ and $\mathbf{H}_{2} \mathrm{BPEF}_{4} \mathbf{B}$ pressed into pellets was estimated by room temperature metal-insulator-metal parallel plate capacitance measurements at $2 \mathrm{~V}$ and in the frequency range 2 $\mathrm{Hz}-2 \times 10^{6} \mathrm{~Hz}$ with an Agilent E4980A Precision LCR Meter connected with an Agilent 16048A Test Leads to a custom-made sample-holder and measuring head (Officina Elettrotecnica di Tenno, Ponte Arche, Italy). 0.5-mm thick, $12-\mathrm{mm}$ wide pellets of $\mathrm{H}_{2} \mathbf{B P E F B}, \mathrm{H}_{2} \mathbf{B P E F}_{2} \mathrm{~B}$ and $\mathrm{H}_{2} \mathrm{BPEF}_{4} \mathrm{~B}$ were prepared by pressing powdered batches of the three materials at $3.3 \mathrm{kbar}$ for $5 \mathrm{~min}$ and were kept in a desiccator prior to measurement. The real component of $\kappa$ was estimated as the ratio between the capacitance of the pellet and that of air, the latter measured after setting the same distance, among the capacitors, at which the capacitance of the pellet was measured. The instrument was calibrated each time it was switched on by measuring the dielectric constant of a 12-mm wide, 1-mm thick pellet of Teflon ${ }^{\text {TM }}$. As a representative example of the calibration procedure, we obtained $\kappa=1.82(3)$ vs. $\kappa=1.9-2.1$ as reported in the literature. ${ }^{66}$ Each measurement, on both Teflon $^{\mathrm{TM}}$ and the title ligands, was repeated five times on the same pellet, alternating one acquisition on the pellet to one on air. The structural integrity of the pressed samples was verified by means of PXRD, as detailed in 2.1. As for the measurement of the dielectric constant after pellet exposure to water vapour, prior to the measurement the pellet was left $24 \mathrm{~h}$ in an air-tight cell at nearly saturated humidity levels.

\subsection{Measurement of the contact angle}

0.5-mm thick pellets of $\mathrm{H}_{2}$ BPEFB and $\mathrm{H}_{2} \mathrm{BPEF}_{2} \mathrm{~B}$ were prepared by pressing powdered batches of the two ligands at $3.3 \mathrm{kbar}$ for 5 min. A drop of distilled water was then laid down the surface. A series of pictures was taken with a common camera and processed by the software Image ${ }^{67}$ to estimate an average value of the contact angle.

\section{Results and Discussion}

\subsection{Ligands synthesis}

The palladium-catalysed Sonogashira cross-coupling reaction is a well-established protocol to form a new carbon-carbon bond. ${ }^{68}$ Due to the deactivating influence of the negatively charged nitrogen atom of the pyrazolate anion on the reactivity of the halogen atom in position 4, special conditions (a large amount of palladium catalyst, $\mathrm{Pd} / \mathrm{C} 10 \%$, and an inorganic base, such as $\mathrm{K}_{2} \mathrm{CO}_{3}$, in dimethyl ether $/ \mathrm{H}_{2} \mathrm{O}$ ) are necessary when $\mathrm{N}$-unsubstituted pyrazoles are involved. ${ }^{69}$ The use of a $N$-protecting group is therefore highly recommended, as it allows performing the Sonogashira reaction under standard conditions and purifying the product with chromatographic techniques. In the present case, ethyl vinyl ether was chosen as protecting agent for the mild conditions involved in both protection and deprotection steps. ${ }^{70}$ In general, the presence of electron withdrawing substituents such as fluorine on the aryl halide species is desirable, as the initial oxidative addition to the $\operatorname{Pd}(0)$ catalytic complex benefits from the presence of electronwithdrawing groups. However, protected 4-ethynylpyrazole has a high tendency to homodimerize under Sonogashira cross-coupling conditions, forcing the use of 4-iodo-(1-ethoxyethyl)pyrazole as starting material. ${ }^{55}$ As reported in Scheme 2, the three protected fluorinated bis(pyrazolyl) intermediates (2a-c) were prepared from their fluorinated $p$-diethynylbenzene precursors (1a-c). Diethynylbenzenes 1a-c were reacted at $343 \mathrm{~K}$ with 4-iodo-(1ethoxyethyl)pyrazole, in the presence of bis(triphenylphosphine)palladium(II) dichloride and copper(I) iodide as catalysts, in a 1:1 mixture of tetrahydrofuran (THF) and 
triethylamine (TEA). 1,4-Bis(1-ethoxyethylpyrazol-4-ylethynyl)-2fluorobenzene (2a), 1,4-bis(1-ethoxyethylpyrazol-4-ylethynyl)-2,3difluorobenzene (2b) and 1,4-bis(1-ethoxyethylpyrazol-4-ylethynyl)tetrafluorobenzene (2c) were purified via flash column chromatography. Purification revealed to be particularly challenging, due to the presence of diethynylbenzene homo-coupling sideproducts with an elution behaviour very similar to that of the desired products. The protected ligands were characterized via ${ }^{1} \mathrm{H},{ }^{13} \mathrm{C}$ and ${ }^{19} \mathrm{~F}$ nuclear magnetic resonance spectroscopy (Figs. S7-S15 of the $\mathrm{ESI}^{\dagger}$ ). The ${ }^{19} \mathrm{~F}$ NMR spectra of $\mathbf{2 a - 2 c}$ (Figs. S9, S12, S15 of the ESI ${ }^{\dagger}$ ) are characterized by the presence of a singlet with a chemical shift ranging from $-110.5 \mathrm{ppm}$ for $\mathbf{2 a}$, to $-138.0 \mathrm{ppm}$ for $\mathbf{2 c}$, as a consequence of the decreasing electron density on the aromatic ring.

In the last step of the synthesis (Scheme 2), the ethoxyethyl protecting groups were removed under mild acidic conditions. To the aim, compounds 2 a-c were dissolved in 1,4-dioxane and reacted at room temperature with small portions of a $6 \mathrm{M}$ aqueous solution of hydrochloric acid. The target ligands 1,4 -bis( $1 \mathrm{H}$-pyrazol-4-ylethynyl)2-fluorobenzene ( $\left.\mathrm{H}_{2} \mathrm{BPEFB}\right), \quad$ 1,4-bis(1H-pyrazol-4-ylethynyl)-2,3difluorobenzene $\left(\mathbf{H}_{2} \mathbf{B P E F}_{2} \mathbf{B}\right)$ and 1,4-bis(1H-pyrazol-4-ylethynyl)tetrafluorobenzene $\left(\mathbf{H}_{2} \mathbf{B P E F}_{4} \mathbf{B}\right)$ were then isolated by filtration. In this case too, the products were characterized $v i a{ }^{1} \mathrm{H},{ }^{13} \mathrm{C}$ and ${ }^{19} \mathrm{~F}$ NMR spectroscopy (Figs. S16-S24 of the ESI ${ }^{\dagger}$ ).

\subsection{Ligands crystal and molecular structure}

Single crystals of quality suitable for an X-ray diffraction structure determination were obtained by room temperature slow evaporation of dimethyl sulfoxide solutions.

$\mathrm{H}_{2} \mathrm{BPEFB}, \mathrm{H}_{2} \mathrm{BPEF}_{2} \mathrm{~B}$ and $\mathrm{H}_{2} \mathrm{BPEF}_{4} \mathrm{~B}$ crystallize in the space group $P 2_{1} / C$, featuring half of the ligand, lying on a crystallographic inversion centre, as the asymmetric unit. The molecular structures of the three ligands are reported in Figs. S25-S27 of the ESI ${ }^{\dagger}$. In $\mathrm{H}_{2}$ BPEFB and $\mathrm{H}_{2} \mathrm{BPEF}_{2} \mathbf{B}$, the central aromatic ring is disordered in two equivalent positions (Fig. S26 of the $\mathrm{ESI}^{+}$), which are related by a pseudo rotation of $180^{\circ}$ around the principal molecular axis. The presence of an increasing number of fluorine atoms on the central aromatic ring has consequences on the molecular conformation adopted by the molecules. Indeed, in $\mathrm{H}_{2}$ BPEFB, the fluorinated moiety is almost coplanar with the pyrazole rings (angle between the r.m.s. planes of the hexa- and penta-atomic rings $=4^{\circ} ;$ Fig. 1). On the other hand, in $\mathbf{H}_{2} \mathbf{B P E F}_{2} \mathbf{B}$ and $\mathbf{H}_{2} \mathbf{B} \mathbf{P E F}_{4} \mathbf{B}$, the dihedral angle between the central ring and the peripheral pyrazole rings is $61^{\circ}$ and $72^{\circ}$, respectively (Fig. 1). As a consequence, despite sharing similar unit cell parameters (Table $\mathrm{S} 1$ of the ESI ${ }^{\dagger}$ ), $\mathrm{H}_{2}$ BPEFB and $\mathrm{H}_{2} \mathrm{BPEF}_{2} \mathrm{~B}$ show different packing.

Fig. 1 The molecular conformations adopted by $\mathrm{H}_{2} \mathrm{BPEFB}, \mathrm{H}_{2} \mathrm{BPEF}_{2} \mathrm{~B}$ and $\mathrm{H}_{2} \mathrm{BPEF}_{4} \mathrm{~B}$ in their crystal structures. Colour code: carbon, grey; hydrogen, white; fluorine, light green; nitrogen, blue.

In $\mathrm{H}_{2}$ BPEFB, by means of hydrogen bonds between the pyrazole

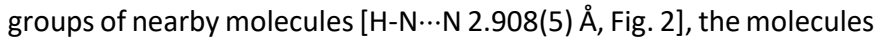
form 2D puckered layers that are approximately parallel to the (-103) crystallographic plane (Fig. 3a).

Fig. 2 The hydrogen bond patterns shown by the pyrazole rings in the crystal structures of the three ligands. Colour code: carbon, grey; hydrogen, white; fluorine, light green; nitrogen, blue.

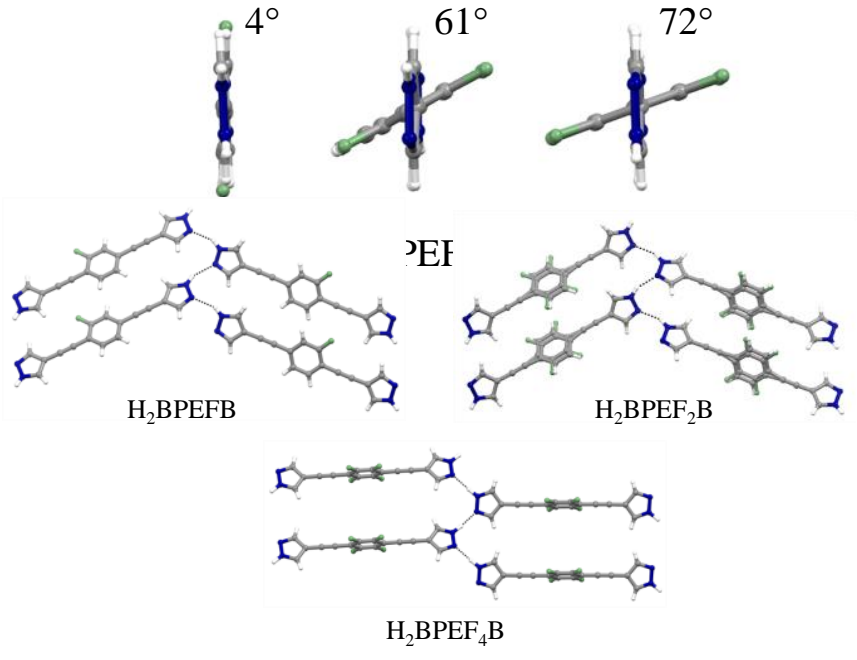

Neighbouring layers form $\mathrm{C}-\mathrm{H} \cdots \mathrm{F}$ and $\mathrm{C}-\mathrm{H} \cdots \pi$ non-bonding

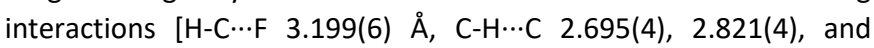
$2.875(4) \AA]$ with symmetry-related molecules (Fig. 3b). A similar

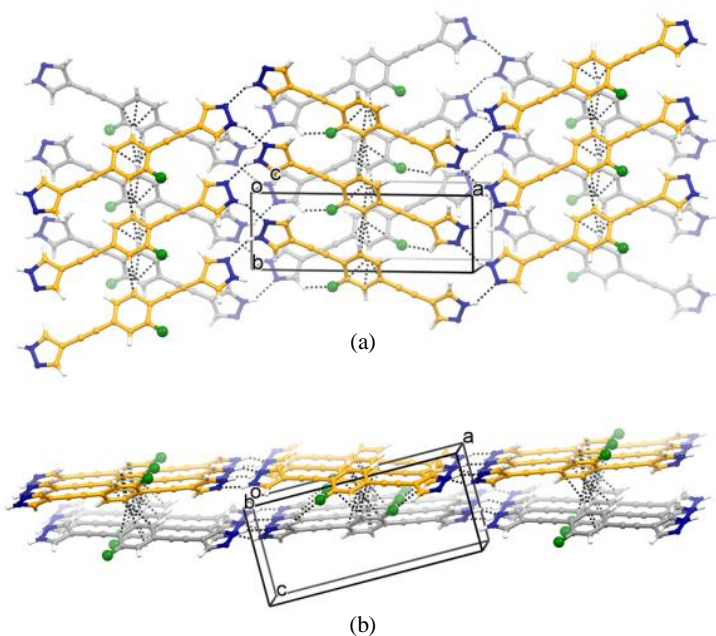

situation is found in $\mathrm{H}_{2} \mathrm{BPEF}_{2} \mathrm{~B}$; also in this case, hydrogen bonds among facing pyrazole rings [H-N N.N 2.867(3) $\AA$, Fig. 2] form 2D puckered layers parallel to the $(-104)$ crystallographic plane (Fig. $\mathrm{S} 28 \mathrm{a}$ of the $\left.\mathrm{ESI}^{+}\right)$. Nearby layers are connected by means of $\mathrm{N} \cdots \mathrm{F}$ non-

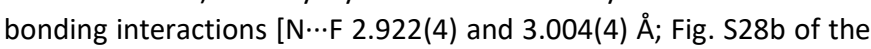
$\left.\mathrm{ESI}^{\dagger}\right]$ and $\pi-\pi$ stacking between the pyrazole and phenyl rings $\left[N_{p 2} \cdots C_{p h} 3.19(1) \AA\right]$. Finally, $\mathbf{H}_{2} B_{P E F_{4}} B$ features hydrogen bonds among facing pyrazole rings [H-N..N 2.938(3) $\AA$, Fig. 2] thus forming 2D layers parallel to the (-101) crystallographic plane (Fig. S29a of the $\left.E S I^{+}\right)$. The fluorine atoms of one layer exchange $F-\pi$ interactions

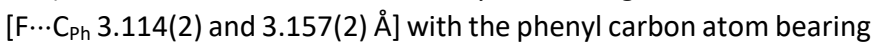
the alkyl moiety of nearby layers. This interaction can be ascribed to the complementary charges possessed by the fluorine atoms (negative) and the aromatic carbon atoms (positive) ${ }^{71}$ which are part of a fluorinated aromatic ring. The $\mathrm{F} 1$ atom is also involved into $\mathrm{C}_{\mathrm{pz}}{ }^{-}$ $\mathrm{H} \cdots \mathrm{F}$ non-bonding interactions with two symmetry related pyrazole rings [H-C...F 3.125(2) and 3.303(2) $\AA$; Fig. S29b of the $\left.\mathrm{ESI}^{\dagger}\right]$.

Fig. 3 Portion of the crystal structure of $\mathbf{H}_{2}$ BPEFB viewed, in perspective, along the crystallographic directions (a) [001] and (b) [010]. The carbon atoms of two consecutive 2-D layers are highlighted in yellow and grey, respectively. Non-bonding 
interactions are represented with dashed lines. Colour code: hydrogen, white; fluorine, green; nitrogen, blue.

A summary of the overall packing motives shown by the three compounds is depicted in Fig. 4. As evident from Fig. 2, the compounds, though having different overall packings, exhibit the same chain-like hydrogen bond pattern, whose graph set can be designed as $\mathrm{C}(3) \mathrm{R}_{4}{ }_{4}(36)$.

Fig. 4 Schematic representation of the molecules' arrangements in the crystal structures of the three ligands.

\subsection{Electronic states transition spectroscopy}

The UV-Vis absorption and fluorescence emission spectra of the three ligands were recorded in both the solid-state and a panel of solvents differing as to their polarity and hydrogen-bonding properties, namely: toluene, chloroform, acetonitrile, tetrahydrofuran, dimethylsulfoxide (DMSO), ethanol, ethyl acetate, and dichloromethane. The fluorescence spectra were recorded also in acetone, where the solvent absorption in the UVB region prevented the UV-Vis spectrophotometric analysis from being undertaken. The solid-state spectra should be compared to that obtained for the non-fluorinated ligand, published in 52. The characterization of the non-fluorinated ligand in solution, which was previously unpublished, was also undertaken for the sake of

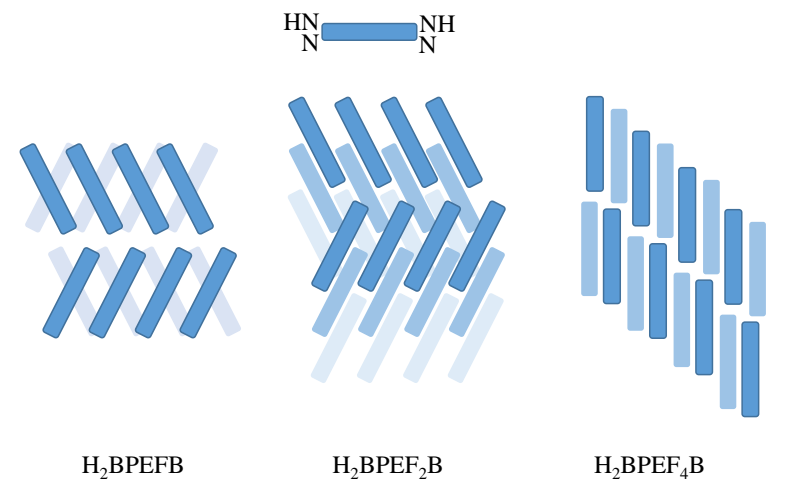

comparison and is reported hereby. In Fig. 5 we report the absorption [panel a)] and emission [panel b)] spectra of the three fluorinated ligands in the solid state. In the Supplementary Material [Fig. S3Oa-c of the $\mathrm{ESI}^{+}$] we provide plots of the absorption and emission spectral line-shape of each compound.
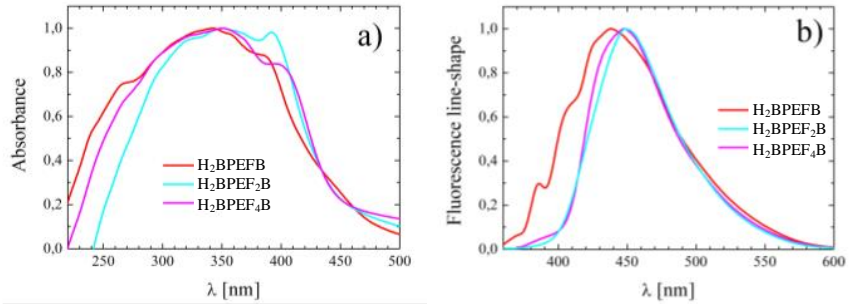

Fig. 5 Panel a): peak-normalized solid-state absorption spectra of $\mathrm{H}_{2}$ BPEFB (red), $\mathrm{H}_{2} \mathrm{BPEF}_{2} \mathrm{~B}$ (cyan), and $\mathrm{H}_{2} \mathrm{BPEF}_{4} \mathrm{~B}$ (magenta). Panel b): peak-normalized solid-state fluorescence spectra of the same samples. Colour codes are conserved.
As for the absorption and fluorescence features of the four ligands in solution, their peak absorption wavelengths in the solvents quoted above are reported as $\lambda_{\text {abs }}$ in the second column of Tables 1 4. The absorption spectral line-shapes for the four compounds in the exemplary solvent acetonitrile are plotted in Fig. 6a. Similar plots obtained in all the other solvents are provided in the Supplementary Material as Figs. S31a-g of the $\mathrm{ESI}^{\dagger}$. Irrespectively of the fluorination degree, all the compounds show a rather structure-less and broadband absorption in the UVB spectral region. Moreover, the absorption spectrum is only slightly dependent on either the solvent or the number of fluorine atoms present in the aromatic ring.
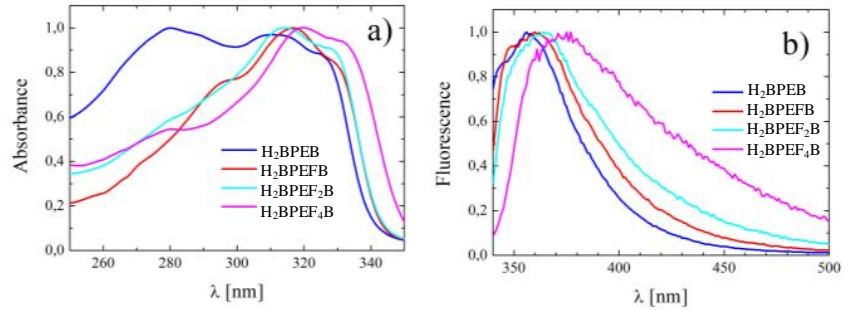

Fig. 6 a) Absorption and b) fluorescence spectra of compounds $\mathrm{H}_{2} \mathrm{BPEB}$ (blue), $\mathrm{H}_{2} \mathrm{BPEFB}$ (red), $\mathrm{H}_{2} \mathrm{BPEF}_{2} \mathrm{~B}$ (cyan), and $\mathrm{H}_{2} \mathrm{BPEF}_{4} \mathrm{~B}$ (magenta) in acetonitrile.

The peak fluorescence wavelengths of $\mathbf{H}_{2} \mathrm{BPEB}, \mathrm{H}_{2} \mathrm{BPEFB}$, $\mathrm{H}_{2} \mathrm{BPEF}_{2} \mathrm{~B}$ and $\mathrm{H}_{2} \mathrm{BPEF}_{4} \mathrm{~B}$ in the solvents quoted above are reported as $\lambda_{\text {fluo }}$ in the third column of Tables 1-4. Shoulders are indicated in parentheses. The pertaining spectral line-shapes for the four compounds in the exemplary solvent acetonitrile are plotted in Fig. $6 \mathrm{~b}$. Similar plots obtained in all the other solvents are given in the Supplementary Material as Figs. S32a-h of the $\mathrm{ESI}^{+}$. With the only exceptions of chloroform and DMSO, the spectral features are poorly sensitive to the environment.

Table 1 Peak absorption wavelengths $\left(\lambda_{\text {abs }}\right)$, peak fluorescence wavelengths ( $\lambda_{\text {fluo }}$ ) and fluorescence quantum yields ( $\phi_{\text {fluo }}$ ) of $\mathrm{H}_{2}$ BPEB in the different solvents essayed. Shoulders are indicated in parenthesis.

\begin{tabular}{lrrr}
\hline Solvent & $\lambda_{\text {abs }}[\mathrm{nm}]$ & $\lambda_{\text {fluo }}[\mathrm{nm}]$ & $\phi_{\text {fluo }}$ \\
\hline Toluene & 317 & 355 & 0.024 \\
Chloroform & 322 & 366 & 0.006 \\
Tetrahydrofuran & 312 & 358 & 0.020 \\
Dichloromethane & 316 & 356 & 0.016 \\
Ethyl Acetate & 311 & 355 & 0.009 \\
Acetone & - & 358 & - \\
Acetonitrile & 311 & 356 & 0.005 \\
Ethanol & 310 & 354 & 0.004 \\
Dimethylsulfoxide & 311 & 365 & 0.013 \\
\hline
\end{tabular}

Table 2 Same data for $\mathrm{H}_{2}$ BPEFB.

\begin{tabular}{lrrr}
\hline Solvent & $\lambda_{\text {abs }}[\mathrm{nm}]$ & $\lambda_{\text {fluo }}[\mathrm{nm}]$ & $\phi_{\text {fluo }}$ \\
\hline Toluene & 320 & $345-362$ & 0.057 \\
Chloroform & 325 & $363-387$ & 0.006 \\
Tetrahydrofuran & 321 & $349-364$ & 0.043
\end{tabular}




\begin{tabular}{lrrr} 
Dichloromethane & 319 & $347-361$ & 0.056 \\
Ethyl Acetate & 319 & $346-361$ & 0.020 \\
Acetone & - & $350-363$ & - \\
Acetonitrile & 317 & $(347)-362$ & 0.013 \\
Ethanol & 318 & $345-361$ & 0.011 \\
Dimethylsulfoxide & 323 & $(355)-370$ & 0.017 \\
\hline
\end{tabular}

Table 3 Same data for $\mathrm{H}_{2} \mathrm{BPEF}_{2} \mathrm{~B}$.

\begin{tabular}{lrrr}
\hline Solvent & $\lambda_{\text {abs }}[\mathrm{nm}]$ & $\lambda_{\text {fluo }}[\mathrm{nm}]$ & $\phi_{\text {fluo }}$ \\
\hline Toluene & 317 & $346-362$ & 0.045 \\
Chloroform & 321 & $359-382$ & 0.007 \\
Tetrahydrofuran & 318 & $350-366$ & 0.015 \\
Dichloromethane & 315 & $350-363$ & 0.019 \\
Ethyl Acetate & 315 & $347-363$ & 0.010 \\
Acetone & - & 364 & - \\
Acetonitrile & 314 & 364 & 0.005 \\
Ethanol & 313 & $348-361$ & 0.006 \\
Dimethylsulfoxide & 323 & 373 & 0.006 \\
\hline
\end{tabular}

Table 4 Same data for $\mathrm{H}_{2} \mathrm{BPEF}_{4} \mathrm{~B}$.

\begin{tabular}{lrrr}
\hline Solvent & $\lambda_{\text {abs }}[\mathrm{nm}]$ & $\lambda_{\text {fluo }}[\mathrm{nm}]$ & $\phi_{\text {fluo }}$ \\
\hline Toluene & 322 & $355-372$ & 0.046 \\
Chloroform & 323 & $357-373$ & 0.042 \\
Tetrahydrofuran & 323 & $361-376$ & 0.006 \\
Dichloromethane & 320 & $358-369$ & 0.034 \\
Ethyl Acetate & 321 & $358-372$ & 0.008 \\
Acetone & - & 375 & - \\
Acetonitrile & 320 & 373 & 0.004 \\
Ethanol & 319 & $358-371$ & 0.007 \\
Dimethylsulfoxide & 328 & 387 & 0.004 \\
\hline
\end{tabular}

The fluorescence quantum yield values were also evaluated by comparison with dimethyl-POPOP in cyclohexane $\left(\Phi_{\text {fluo }}=0.96\right)$, as described in detail elsewhere. ${ }^{72}$ The quantum yields, reported in the fourth column of Tables 1-4, do not exhibit a straightforward trend with either solvent polarity or fluorination degree. This occurrence, together with the environment-independent spectral features, might be beneficial in view of application to VOCs sensing, as the fluorescence signal is expected to be robust with respect to environmental perturbations other than $\pi-\pi$ stacking with the VOCs aromatic ring, including self-assembly within a MOF. Moreover, although the quantum yield values are generally quite low, in the range of a few percent at most, fluorination does not result in fluorescence quenching. It is worth noting that the fluorescence signal of fluorophores with such quantum yields, emitting in the UVBto-blue spectral band upon excitation with low-cost incoherent light sources (e.g.: a suitably filtered Wood lamp), can be easily detected by means of low-cost solid-state detectors (e.g.: silicon photomultipliers).

Further discussions on the spectroscopic data will be undertaken in light of the in-silico results reported in Section 3.4.

\subsection{Electronic structure modelling}

Spectroscopy. The TD-DFT results suggest that only the electronic excitations involving the HOMO and LUMO of the four ligands should provide an active transition when the excitation wavelength is above
$260 \mathrm{~nm}$. In this respect, Table 2 presents the wavelength of such transitions for the four systems considered, while Fig. 7 shows, as a representative example, the two molecular orbitals (MO's) involved in the case of the $\mathrm{H}_{2}$ BPEB ligand.

Apart from the evident (and commonly found) red-shift of the theoretical values in Table 5 with respect to the experimental data in non-polar solvents (Tables 1-4), we notice that TD-DFT closely mimics the slight bathochromic shift induced by the hydrogen-fluorine substitution observed in the latter. The symmetrical shapes of the two frontier MO's (Fig. 7) make also apparent that the excitation should not involve any relevant charge transfer, a finding that rationalizes the limited impact that a change in the dielectric constant of the solvents employed to collect the absorption spectra has on the location of the absorption peak. This notwithstanding, it is worth noticing that the spectra collected in chloroform and dimethylsulphoxide deviate somewhat from this general rule, the absorption peak measured in these two solvents being apparently red-shifted by roughly $3-5 \mathrm{~nm}$ with respect to those detected in the other solvents (vide infra for further discussion).

Table 5 Wavelength and photon energy for the gas-phase excitation involving the active HOMO-LUMO transition in the four ligands.

\begin{tabular}{lcr}
\hline Ligand & $\lambda[\mathrm{nm}]$ & Photon energy [eV] \\
\hline $\mathrm{H}_{2} \mathrm{BPEB}$ & 343.97 & 3.6045 \\
$\mathrm{H}_{2} \mathrm{BPEFB}$ & 347.06 & 3.5724 \\
$\mathrm{H}_{2} \mathrm{BPEF}_{2} \mathrm{~B}$ & 347.38 & 3.5691 \\
$\mathrm{H}_{2} \mathrm{BPEF}_{4} \mathrm{~B}$ & 353.16 & 3.5107 \\
\hline
\end{tabular}

The lack of strongly absorbing transitions other than the one involving the HOMO and LUMO indicated by the TD-DFT results suggests that the shape of the absorption bands at shorter wavelengths with respect to the lowest energy feature in, e.g., Fig. 6 ought to be mainly due to transitions involving also the vibrational excitation of the electronically excited species. To support this idea, we optimized the structure of the $\mathbf{H}_{2} \mathbf{B P E B}$ ligand on the potential energy surface of its lowest electronic excited state in order to obtain the vibrational frequencies. The latter evidenced the presence of five intense vibrational excitations [ $\mathrm{v}\left(\mathrm{cm}^{-1}\right), \mathrm{I}(\mathrm{km} / \mathrm{mol}): 1154,810 ; 1253$, $740 ; 1424,844 ; 1471,379 ; 1513,243]$ involving the bending motion of hydrogen atoms located on pyrazole or phenyl rings, which, alone or in combination, may explain the location of the features evident in Fig. 6.

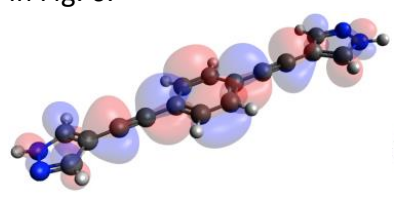

(a)

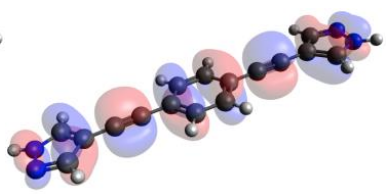

(b)
Fig. 7 (a) HOMO and (b) LUMO for the ligand $\mathbf{H}_{2} B P E B$, whose shape closely resembles that of the two frontier molecular orbitals for the $\mathrm{H}_{2} \mathrm{BPEFB}, \mathrm{H}_{2} \mathrm{BPEF}_{2} \mathrm{~B}$, and $\mathrm{H}_{2} \mathrm{BPEF}_{4} \mathrm{~B}$ ligands.

From the optimized structure of the $\mathrm{H}_{2}$ BPEB ligand on the potential energy surface of its lowest excited state, one may also extract the energy of the photon released upon fluorescent 
relaxation of the excited system assuming its vertical nature. In such case, the emitted photon should have a wavelength of $381.5 \mathrm{~nm}$ $(3.250 \mathrm{eV})$, which is $10 \%$ lower than the one predicted for the vertical excitation (see Table 5). Compared to the experimental data on $\mathbf{H}_{2} \mathbf{B P E B}$, the theoretical result appears substantially red-shifted (roughly, by $15-25 \mathrm{~nm}$ ); the difference between absorbed and emitted photon energies is instead rather well predicted by TD-DFT, the results from the latter $(0.3551 \mathrm{eV})$ differing by only $0.046 \mathrm{eV}$ from the experimentally estimated one $(0.309 \mathrm{eV})$.

The possible origin of the bathochromic shift of the electronic absorption evidenced for the ligands when dissolved in chloroform and dimethylsulfoxide was also investigated by explicitly adding a solvent molecule to the $\mathrm{H}_{2} \mathrm{BPEB}$ chromophore and re-computing the excitation energies following geometry optimization. The structure of the optimized dimers is shown in Fig. 8. From the latter, it can be noticed that the dominant interaction in both cases seems to be of the dipole-dipole type, evidenced by the formation of hydrogen bonds. In particular, chloroform forms two hydrogen bonds (one donated, $\mathrm{C}-\mathrm{H} \cdots \mathrm{N}$, and one accepted, $\mathrm{N}-\mathrm{H} \cdot \cdots \mathrm{Cl}$ ), while the dimethylsulfoxide oxygen acts as acceptor of a single hydrogen bond from the $\mathrm{N}-\mathrm{H}$ group on the pyrazole ring. As for the transition energies, we have found that the HOMO-LUMO excitations are either red-shifted when dimethylsulfoxide is present $(\Delta \lambda=2.8 \mathrm{~nm})$, or substantially unmodified $(\Delta \lambda=-0.2 \mathrm{~nm}$ ) in presence of chloroform. $A$ possible rationale for such differences may be provided by the fact that, while the LUMO of $\mathbf{H}_{2}$ BPEB is left largely unaffected by the solvation with chloroform, thus maintaining an extremely limited participation of the $p$ orbital on the nitrogen atom $\left(p_{\mathrm{N} 2}\right)$ in position 2 to this $\mathrm{MO}$, it appears to have a sizable component from the same atomic orbital when DMSO is involved. Clearly, the mixing of the $p_{\mathrm{N} 2}$ orbital must descend from a modification of its average energy due to a change in its molecular environment, which is represented in this case by the presence of two weakly polar $\mathrm{C}-\mathrm{H}$ bonds pointing toward it (Fig. 8), and suggests that a lowering of the LUMO eigenvalue ought to be expected due to the widening of its shape (i.e. a lower kinetic energy).

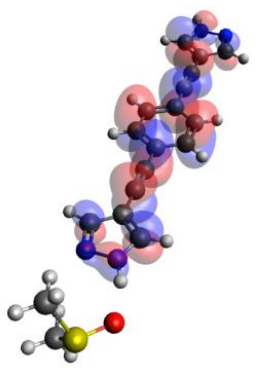

(a)

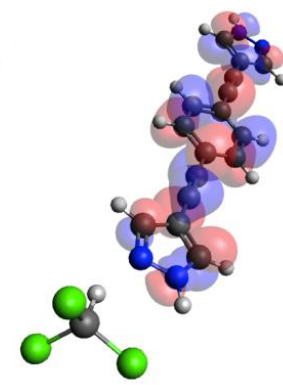

(b)
Fig. 8 Minimum energy structure for the dimers involving $\mathbf{H}_{2} \mathbf{B P E B}$ interacting with (a) dymethylsulphoxide or (b) chloroform. The LUMO for both dimers is also shown.

Intermolecular interactions. The molecular organization in space of ligands such as $\mathrm{H}_{2} \mathrm{BPEB}$ when forming MOFs with late transition metal ions $[\mathrm{Zn}(\mathrm{II}), \mathrm{Ni}(\mathrm{II}) \text {, or } \mathrm{Fe}(\mathrm{III})]^{52}$ allows the atoms composing the phenyl ring to interact directly with small molecular gasses (e.g. water or $\mathrm{CO}_{2}$ ) adsorbed inside the porous materials. It thus becomes interesting to gauge how intermolecular interactions between possible adsorbed species and $\mathbf{H}_{2} \mathbf{B P E F}_{\mathbf{X}} \mathbf{B}(\mathbf{X}=0,1,2,4)$ are modified by the fluorination as it may change, e.g., the relative preference when competitive adsorption experiments are executed. Exploiting the presence of strong multipolar moments, we have thus employed DFT calculations at the $B 3 L Y P / 6-31++G(d, p)$ level to optimize minimum energy structures for dimers composed of a $\mathbf{H}_{2} \mathbf{B} P \mathbf{F}_{\mathbf{X}} \mathbf{B}(\mathrm{X}=$ 0 or 4) species and either water or $\mathrm{CO}_{2}$. Apart from issues related to the computational cost of finding all minimum energy structures in low symmetry molecules, the motivation for investigating ligands with $X=0$ or 4 descends from the intention of maximizing the effect of substitution on the electron density of the ligands.

Fig. 9 shows the minimum energy structure obtained for the four possible dimers, together with the difference in total energy between the complex and the asymptotically distant fragments; notice that we were able to locate two minima for the species $\mathrm{H}_{2} \mathrm{BPEF}_{4} \mathrm{~B}-\mathrm{H}_{2} \mathrm{O}$.

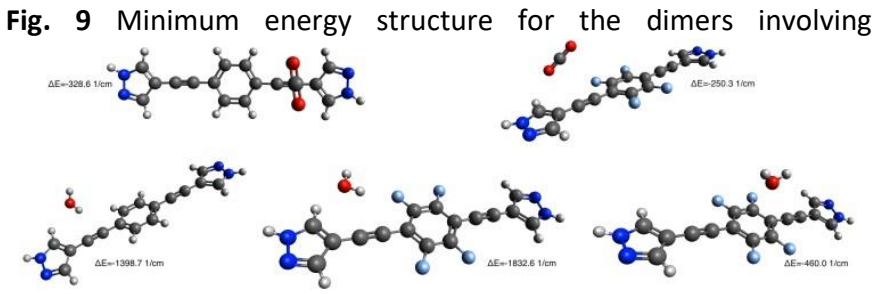

$\mathrm{H}_{2} \mathrm{BPEFB} / \mathrm{H}_{2} \mathrm{BPEF}_{4} \mathrm{~B}$ interacting with water or carbon dioxide. $\triangle \mathrm{E}$ indicates the energy difference between the shown systems and the asymptotic fragments.

Considering only the lowest lying geometry for each of the possible dimers, we notice that fluorination has opposite impacts on the interaction energy for water and carbon dioxide. Thus, while it increases $\Delta \mathrm{E}$ by $78.3 \mathrm{~cm}^{-1}$ in the case of $\mathrm{CO}_{2}$, it deepens the well by $433.9 \mathrm{~cm}^{-1}$ when water is involved. From the structures obtained, it is apparent that the opposite behaviours may be easily rationalized basing on the different interaction modalities. Thus, the markedly dipolar nature of water and its ability to form hydrogen bonds with any dense electron source available (i.e. triple bonds or fluorine) makes it capable of exploiting any change in electron density by switching between possible interacting groups, whereas carbon dioxide suffers from the electron attracting effect of fluorine that lowers the electron density of the triple bond. Noteworthy, there is also the fact that the second minimum found for $\mathrm{H}_{2} \mathrm{BPEF}_{4} \mathrm{~B}-\mathrm{H}_{2} \mathrm{O}$ has an interaction energy nearly twice as large as the one for $\mathbf{H}_{2} \mathbf{B P E F}_{4} \mathbf{B}$ $\mathrm{CO}_{2}$, de facto making the adsorption of the latter gas on the tetrafluorinated species not competitive with respect to water.

Albeit the electron withdrawing effect that fluorine has on the phenyl ring and, possibly, on the conjugated triple bonds works against the usage of the fluorinated ligands as selective adsorbents for carbon dioxide when water vapor is present, they may instead be exploited to foster adsorption of aromatic species with sizes compatible with MOFs pores. To check if this is the case, we investigated the interaction energy between toluene and the same two $\mathrm{H}_{2} \mathrm{BPEF}_{\mathbf{X}} \mathrm{B}$ ( $\mathrm{X}=0$ or 4 ) species by optimizing the dimers structure to obtain the relevant well depths. Due to the relative importance that London-type interactions may have when conjugated systems 
interact, a second order Møller-Plesset (MP2) perturbation theory level was employed in conjunction with the $6-31++G(d, p)$ basis set. Besides, interaction energies have been corrected for possible basis set superposition error (BSSE) via the Counterpoise (CP) methods of Boys and Bernardi. ${ }^{65} \mathrm{Fig} .10$ shows the two stationary points together with their energetics relative to the asymptotically distant fragments.
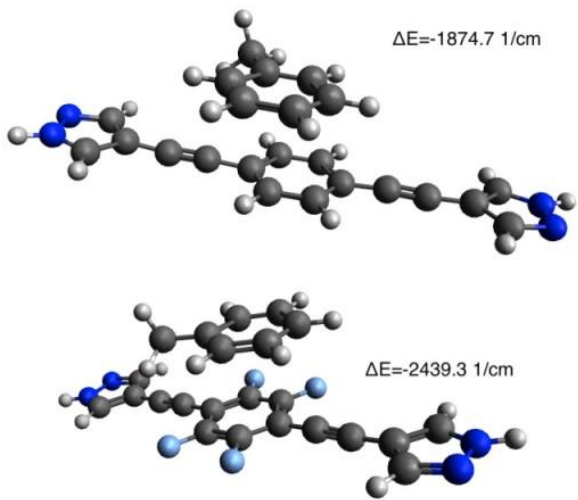

Fig. 10 Minimum energy structure for the dimers involving $\mathrm{H}_{2} \mathrm{BPEFB} / \mathrm{H}_{2} \mathrm{BPEF}_{4} \mathrm{~B}$ interacting with toluene. $\triangle \mathrm{E}$ indicates the energy difference between the shown systems and the asymptotic fragments.

From the structures shown, it clearly emerges that the interaction modality with toluene is similar for both ligands, the aromatic molecule laying parallel to the phenyl ring in the ligands, but with its geometrical centre being shifted sideways in order to minimize the overlap between the clouds of the $\pi$ systems. As for the interaction energy, it is evident that fluorinating may have a beneficial effect on the adsorption of toluene, as the interaction well for the fluorinated dimer is roughly $565 \mathrm{~cm}^{-1}$ deeper than the one for the fully hydrogenated species. Assuming for the sake of simplicity that differences in thermal and entropic effects between the two species are minimal, the adsorption constant of toluene on $\mathrm{H}_{2} \mathrm{BPEF}_{4} \mathrm{~B}$ may be up to 15 times higher than on $\mathbf{H}_{2} \mathbf{B P E B}$ at room temperature.

\subsection{Estimation of the dielectric constant and of the contact angle}

The value of the dielectric constant $(\kappa)$ of $\mathrm{H}_{2}$ BPEFB, $\mathrm{H}_{2} \mathrm{BPEF}_{2} \mathrm{~B}$ and $\mathrm{H}_{2} \mathrm{BPEF}_{4} \mathbf{B}$ pressed into pellets was estimated at room temperature by metal-insulator-metal parallel plate capacitance measurements at $2 \mathrm{~V}$ and in the frequency range $2 \mathrm{~Hz}-2 \times 10^{6} \mathrm{~Hz}$. The results are collected in Table 6, while the curves showing the behaviour of $\kappa$ vs. frequency are reported in Fig. 11. After pellet preparation, the structural integrity of the three ligands was checked by means of PXRD. As shown by Fig. S33 of the ESI ${ }^{\dagger}$, pellettization brings about a moderate loss of crystallinity, but neither amorphization, nor phase change.

Table 6 Values of $\kappa$ at $2 \mathrm{~V}$ and $2 \times 10^{6} \mathrm{~Hz}$ for $\mathrm{H}_{2}$ BPEFB, $\mathrm{H}_{2}$ BPEFB and $\mathrm{H}_{2} \mathrm{BPEF}_{4} \mathrm{~B}$ under ambient and near saturated humidity levels. No data are available for $\mathbf{H}_{2} \mathbf{B P E F}_{4} \mathbf{B}$ after exposure to water vapor due to shortage of sample.

\begin{tabular}{lrr}
\hline Ligand & $\begin{array}{r}\kappa \text { at } 2 \times 10^{6} \mathrm{~Hz} \\
\text { (ambient } \mathrm{RH})\end{array}$ & $\begin{array}{r}\kappa \text { at } 2 \times 10^{6} \mathrm{~Hz} \\
(\sim \text { saturated } \mathrm{RH})\end{array}$ \\
\hline
\end{tabular}

\begin{tabular}{lrr}
\hline H$_{2}$ BPEFB & Ave $=1.94 \pm 0.40$ & Ave $=2.61 \pm 0.26$ \\
& & Min $=2.40$ \\
Max $=3.04$ \\
\hline \\
$\mathrm{H}_{2} \mathrm{BPEF}_{2} \mathrm{~B}$ & Ave $=2.18 \pm 0.10$ & Ave $=5.56 \pm 1.82$ \\
& & Min $=4.40$ \\
& Max $=8.74$ \\
\hline $\mathrm{H}_{2} \mathrm{BPEF}_{4} \mathrm{~B}$ & Ave $=3.00 \pm 0.07$ & n.a. \\
\hline
\end{tabular}

$\mathrm{H}_{2} \mathrm{BPEFB}, \mathrm{H}_{2} \mathrm{BPEF}_{2} \mathrm{~B}$ and $\mathrm{H}_{2} \mathrm{BPEF}_{4} \mathrm{~B}$ show average $\kappa$ values of 1.94(40), $2.18(10)$ and 3.00(7), respectively (with best values of $1.89,2.03$ and 2.91 , respectively), denouncing an increase of the dielectric constant upon increasing the number of fluorine atoms on the central aromatic ring. This occurrence can be tentatively ascribed to the higher number of electrons per molecule provided by the fluorine atoms, which increases the molecule polarizability.

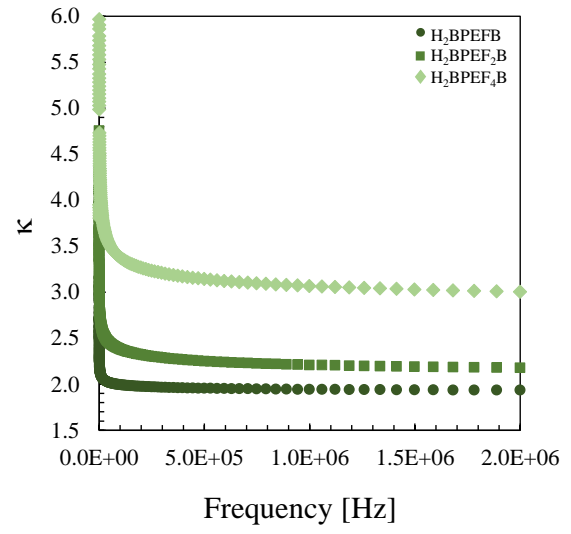

Fig. 11 Behaviour of the dielectric constant as a function of the $A C$ frequency for $\mathbf{H}_{2} \mathrm{BPEFB}$ (dark green circles), $\mathbf{H}_{2} \mathrm{BPEF}_{2} \mathbf{B}$ (green squares) and $\mathbf{H}_{2} \mathbf{B P E F}_{4} \mathbf{B}$ (light green diamonds).

The dielectric constant was also measured after exposure of the pellet to a nearly saturated water vapor environment for 24 hours at room temperature. No data are available in this case for $\mathbf{H}_{2} \mathbf{B P E F}_{4} \mathbf{B}$ due to sample shortage. In both cases, and particularly for $\mathrm{H}_{2} \mathbf{B P E F}_{2} \mathrm{~B}$, the average value of $\kappa$ increases (Table 6 ), indicating that a number of water molecules $(\kappa \sim 80)$ have been adsorbed on the pellets surface. Notably, as witnessed by the maximum and minimum values of $\kappa$ recorded at $2 \times 10^{6} \mathrm{~Hz}$ (Table 6) there is a progressive decrease of $\kappa$ along the five consecutive repetitions of the measurement (Fig. S34 of the $\mathrm{ESI}^{+}$), suggesting a progressive release of the adsorbed water molecules, more pronounced in the case of $\mathrm{H}_{2} \mathrm{BPEFB}$, again pointing toward a non-negligible role of the molecules polarizability. The higher increase of $\kappa$ upon water vapor exposure for $\mathbf{H}_{2} \mathbf{B P E F}_{2} \mathbf{B}$ is in line with the hydrophilicity trends disclosed by the theoretical calculations.

To gain preliminary insight on the hydrophobicity of $\mathrm{H}_{2}$ BPEFB and $\mathrm{H}_{2} \mathrm{BPEF}_{2} \mathbf{B}$, we estimated their static contact angle, as detailed in the Experimental Section. Fig. 12 shows, as a representative example, two of the pictures taken and processed for the estimation. With average contact angle values of $78(1)^{\circ}$ and $84(1)^{\circ}$, lower than $90^{\circ}$, none of the ligands can be strictly classified as hydrophobic. ${ }^{73}$ 
Fig. 12 Estimation of the contact angle for (a) $\mathrm{H}_{2}$ BPEFB and (b) $\mathrm{H}_{2} \mathrm{BPEF}_{2} \mathbf{B}$. One of the images processed for each ligand is shown as a representative example.

\section{Conclusions}

The new fluorinated bis(pyrazoles) 1,4-bis(1H-pyrazol-4-ylethynyl)-2fluorobenzene ( $\mathrm{H}_{2}$ BPEFB), 1,4-bis(1H-pyrazol-4-ylethynyl)-2,3difluorobenzene $\left(\mathrm{H}_{2} \mathrm{BPEF}_{2} \mathrm{~B}\right)$ and 1,4-bis(1H-pyrazol-4-ylethynyl)tetrafluorobenzene $\left(\mathbf{H}_{2} \mathbf{B P E F}_{4} \mathbf{B}\right)$ have been synthesized through Sonogashira coupling reactions, and characterized as per their crystal and molecular structure, solid-state and solution UV-Vis absorption and fluorescence emission, dielectric properties and hydrophobicity. Theoretical modelling unravelled a pronounced affinity of the compounds towards toluene, which notably increases at increasing fluorination. This feature might be exploited in the future for the synthesis of MOFs which could be applied as molecular sieves for the sequestration of this as well as other chemically related VOCs from aqueous environments. Indeed, fluorescent MOF structures were previously obtained with the closely related ligand $\mathbf{H}_{2} \mathbf{B P E B}$. Moreover, the absorption and emission properties depend only slightly on either the solvent or the fluorination degree. These features, together with a sizeable and environment-independent fluorescence quantum yield in the blue-violet portion of the spectrum, where low-cost solid state detectors exhibit their peak detection quantum efficiencies, make the compounds credible candidates as building blocks for luminescent MOFs for VOCs sequestration and luminescence-based sensing.

\section{Conflicts of interest}

There are no conflicts of interest to declare.

\section{Acknowledgements}

This work has been partially funded through the PRIN 20173L7W8K grant by the Italian Ministry of Instruction, University and Research (MIUR). L.N. acknowledges University

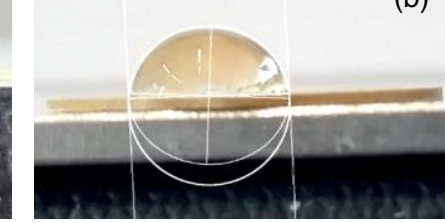

of Insubria for a post-doctorate grant (DISAT2019-ADRJ003). S.G. deeply acknowledges Rebecca Vismara (University of Insubria) for experimental help. The Authors are grateful to Prof. G. Palmisano and S. Tollari (University of Insubria) for fruitful discussions. 
1 X. H. Liu, W. Zhao, Z. H. Shen, J. H. Xing, J. Yuan, G. Yang, T. M. $\mathrm{Xu}$ and W. L. Peng, Synthesis, nematocidal activity and docking study of novel chiral 1-(3-chloropyridin-2-yl)-3(trifluoromethyl)-1H-pyrazole-4-carboxamide derivatives, Bioorg. Med. Chem. Lett., 2016, 26, 3626-3628.

2 S. Güniz Küçükgüzel and S. Senkardes, Recent advances in bioactive pyrazoles, Eur. J. Med. Chem., 2015, 97, 786-815.

3 A. Jamwal, A. Javed and V. Bhardwaj, A review on pyrazole derivatives of pharmacological potential, J. Pharm. BioSci., 2013, 3, 114-123.

4 A. N. Chermahini, A. Teimour, A. S. Beni and F. Dordahan, Theoretical studies on the effect of substituent in the proton transfer reaction of 4-substituted pyrazoles, Comp. Theor. Chem., 2013, 1008, 67-73.

5 S. Mert, R. Kasımogulları, T. Iça, F. Çolak, A. Altun, and S. Ok, Synthesis, structure-activity relationships, and in vitro antibacterial and antifungal activity evaluations of novel pyrazole carboxylic and dicarboxylic acid derivatives, Eur. J. Med. Chem., 2014, 78, 86-96.

6 X. R. Liu, H. Wu, Z. Y. He, Z. Q. Ma, J. T. Feng and X. Zhang, Design, synthesis and fungicidal activities of some novel pyrazole derivatives, Molecules, 2014, 19, 14036-14051.

7 J. X. Mu, J. X. Shi, M. Y. Yang, Z. H. Sun, X. H. Liu, B. J. Li and N. $B$. Sun, Design, synthesis, DFT study and antifungal activity of pyrazolecarboxamide derivatives, Molecules, 2016, 21, 68-79.

8 W. Zhao, J. Xing, T. Xu, W. Peng and X. Liu, Synthesis and in vivo nematocidal evaluation of novel 3-(trifluoromethyl)-1Hpyrazole-4-carboxamide derivatives, Front. Chem. Sci. Eng., 2017, 11, 363-368.

9 C. Pettinari, N. Masciocchi, L. Pandolfo and D. Pucci, Tuning the functional properties of metal complexes containing polytopic heteroaromatic nitrogen ligands, Chem. Eur. J., 2010, 16, 11061123.

10 J. Wu, B. A. Song, D. H. Hu, M. Yue and S. Yang, Design, synthesis and insecticidal activities of novel pyrazole amides containing hydrazone substructures, Pest. Manag. Sci., 2012, 68, 801-810.

11 C. Fu, J. Pei, Y. Ning, M. Liu, P. Shan, J. Liu, Y. Li, F. Hu, Y. Zhu, H. Yang and $X$. Zou, Synthesis and insecticidal activities of novel pyrazole oxime ether derivatives with different substituted pyridyl rings, Pest. Manag. Sci., 2014, 70, 1207-1214.

12 T. Eicher, S. Hauptmann and A. Speicher, The chemistry of heterocycles: structure, reactions, synthesis and applications, Wiley-VCH: 2012.
13 M. A. Halcrow, Pyrazoles and pyrazolides-flexible synthons in self-assembly, Dalton Trans., 2009, 2059-2073.

14 G. Noël, J. C. Röder, S. Dechert, H. Pritzkow, L. Bolk, S. Mecking and F. Meyer, Pyrazolate-based dinuclear $\alpha$-diimine-type palladium(II) and nickel(II) complexes - a bimetallic approach in olefin polymerisation, Adv. Synth. Catal., 2006, 348, 887897.

15 L. Francàs, C. Richmond, P. Garrido-Barros, N. Planas, S. Roeser, J. Benet-Buchholz, L. Escriche, X. Sal and A. Llobet, Rubis(pyridine)pyrazolate (bpp)-based water-oxidation catalysts anchored on $\mathrm{TiO}_{2}$ : the importance of the nature and position of the anchoring group, Chem. Eur. J., 2016, 22, 5261-5268.

16 C. J. Richmond and A. Llobet, Incorporation of a rutheniumbis(pyridine)pyrazolate (Ru-bpp) water oxidation catalyst in a hexametallic macrocycle, Catal. Sci. Technol., 2016, 6, 66976704.

17 F. K. Keter and J. Darkwa, Perspective: the potential of pyrazole-based compounds in medicine, BioMetals, 2012, 25, 9-21.

18 C. Pettinari, A. Tăbăcaru and S. Galli, Coordination polymers and metal-organic frameworks based on poly(pyrazole)containing ligands, Coord Chem Rev., 2016, 307, 1-31.

19 S. Fustero, M. Sánchez-Roselló, P. Barrio and A. Simón-Fuentes, From 2000 to mid-2010: a fruitful decade for the synthesis of pyrazoles, Chem Rev., 2011, 111, 6984-7034.

20 J. Li, X. Wang, G. Zhao, C. Chen, Z. Chai, A. Alsaedi, T. Hayat and $X$. Wang, Metal-organic framework-based materials: superior adsorbents for the capture of toxic and radioactive metal ions, Chem. Soc. Rev., 2018, 47, 2322-2356.

21 H. Li, K. Wang, Y. Sun, C. T. Lollar, J. Li and H.-C. Zhou, Recent advances in gas storage and separation using metal-organic frameworks, Materials Today, 2018, 21, 108-121.

22 N. S. Bobbitt, M. L. Mendonca, A. J. Howarth, T. Islamoglu, J. T. Hupp, O. K. Farha and R. Q. Snurr, Metal-organic frameworks for the removal of toxic industrial chemicals and chemical warfare agents, Chem. Soc. Rev., 2017, 46, 3357-3385.

23 K. Adil, Y. Belmabkhout, R. S. Pillai, A. Cadiau, P. M. Bhatt, A. H. Assen, G. Maurin and M. Eddaoudi, Gas/vapour separation using ultra-microporous metal-organic frameworks: insights into the structure/separation relationship, Chem. Soc. Rev., 2017, 46, 3402-3430.

24 M. Liu, J. Wu and H. Hou, Metal-organic framework (MOF)based materials as heterogeneous catalysts for $\mathrm{C}-\mathrm{H}$ bond activation, Chem. Eur. J., 2019, 25, 2935-2948. 
41 K. S. Park, Z. Ni, A. P. Côté, J. Y. Choi, R. Huang, F. J. Uribe-Romo, H. K. Chae, M. O'Keeffe and O. M. Yaghi, . Exceptional chemical and thermal stability of zeolitic imidazolate frameworks, Proc. Nat. Acad. Sci., 2006, 103, 10186-10191.

42 M. Eddaoudi, J. Kim, N. Rosi, D. Vodak, J. Wachter, M. O'Keeffe and O. M. Yaghi, Systematic design of pore size and functionality in isoreticular MOFs and their application in methane storage, Science, 2002, 295, 469-472.

43 S.-I. Noro and T. Nakamura, Fluorine-functionalized metalorganic frameworks and porous coordination polymers, NPG Asia Materials, 2017, 9, 433.

25 W.-G. Cui, G.-Y. Zhang, T.-L. Hua and X.-H. Bu, Metal-organic framework-based heterogeneous catalysts for the conversion of $\mathrm{C} 1$ chemistry: $\mathrm{CO}, \mathrm{CO}_{2}$ and $\mathrm{CH}_{4}$, Coord. Chem. Rev., 2019, 387, 79-120.

26 A. Dhakshinamoorthy, Z. Li, and H. Garcia, Catalysis and photocatalysis by metal organic frameworks, Chem. Soc. Rev., 2018, 47, 8134-8172.

27 L. Zhu, X.-Q. Liu, H.-L. Jiang and L.-B. Sun, Metal-organic frameworks for heterogeneous basic catalysis, Chem. Rev., 2017, 117, 8129-8176.

28 S. M. J. Rogge, A. Bavykina, J. Hajek, H. Garcia, A. I. OlivosSuarez, A. Sepúlveda-Escribano, A. Vimont, G. Clet, P. Bazin, F. Kapteijn, M. Daturi, E. V. Ramos-Fernandez, F. X. Llabrés i Xamena, V. Van Speybroeck and J. Gascon, Metal-organic and covalent organic frameworks as single-site catalysts, Chem. Soc. Rev., 2017, 46, 3134-3184.

29 A. H. Chughtai, N. Ahmad, H. A. Younus, A. Laypkov and F. Verpoort, Metal-organic frameworks: versatile heterogeneous catalysts for efficient catalytic organic transformations, Chem. Soc. Rev., 2015, 44, 6804-6849.

30 C. Lucarelli, S. Galli, A. Maspero, A. Cimino, C. Bandinelli, A. Lolli, J. Velasquez Ochoa, A. Vaccari, F. Cavani and S. Albonetti, Adsorbent-adsorbate interactions in the oxidation of $\mathrm{HMF}$ catalyzed by Ni-based MOFs: A DRIFT and FT-IR insight, J. Phys. Chem. C, 2016, 120, 15310-15321.

31 W-T. Koo, J.-S. Jang, and I.-D. Kim, Metal-organic frameworks for chemiresistive sensors, Chem., 2019, 5, 1938-1963.

32 Y. Zhang, S. Yuan, G. Day, X. Wang, X. Yang and H.-C. Zhou, Luminescent sensors based on metal-organic frameworks, Coord. Chem. Rev., 2018, 354, 28-45.

33 W. P. Lustig, S. Mukherjee, N. D. Rudd, A. V. Desai, J. Li and S. K. Ghosh, Metal-organic frameworks: functional luminescent and photonic materials for sensing applications, Chem. Soc. Rev., 2017, 46, 3242-3285.

34 F.-Y. Yi, D. Chen, M.-K. Wu, L. Han and H.-L. Jiang, Chemical sensors based on metal-organic frameworks, ChemSusChem., 2016, 81, 675-690.

35 V. Stavila, A. A. Talin and M. D. Allendorf, MOF-based electronic and optoelectronic devices, Chem. Soc. Rev., 2014, 43, 59946010.

36 L. E. Kreno, K. Leong, O. K. Farha, M. D. Allendorf, R. P. Van Duyne and J. T. Hupp, Chem. Rev., Metal-organic framework materials as chemical sensors, 2012, 112, 1105-1125.

37 F. Millange and R. I. Walton, MIL-53 and its isoreticular analogues: a review of the chemistry and structure of a prototypical flexible metal-organic framework, Isr. J. Chem., 2018, 58, 1019-1035.

38 Z.-J. Lin, J. Lü, M. Hong and R. Cao, Metal-organic frameworks

12 I Name., 2012 flexible ligands (FL-MOFs): structures and applications, Chem. Soc. Rev., 2014, 43, 5867-5895.

44 C. Serre, Superhydrophobicity in highly fluorinated porous metal-organic frameworks, Angew. Chem. Int. Ed., 2012, 51, 6048-6050.

45 C. Yang, U. Kaipa, Q. Z. Mather, X. Wang, V. Nesterov, A. F. Venero and M. A. Omary, Fluorous metal-organic frameworks with superior adsorption and hydrophobic properties toward oil spill cleanup and hydrocarbon storage, J. Am. Chem. Soc., 2011, 133, 18094-18097.

46 C. Yang, X. Wang and M. A. Omary, Fluorous metal-organic frameworks for high-density gas adsorption, J. Am. Chem. Soc., 2007, 129, 15454-15455.

47 T.-H. Chen, I. Popov, O. Zenasni, O. Daugulis and O. Š. Miljanić, Superhydrophobic perfluorinated metal-organic Frameworks, Chem. Commun., 2013, 49, 6846-6848.

48 M. Weck, A. R. Dunn, K. Matsumoto, G. W. Coates, E. B. Lobkovsky and R. H. Grubbs, Influence of perfluoroarene-arene interactions on the phase behavior of liquid crystalline and polymeric materials, Angew. Chem. Int. Ed., 1999, 38, 27412745.

49 S. Galli, A. Cimino, J. F. Ivy, C. Giacobbe, R. K. Arvapally, R. Vismara, S. Checchia, M. A. Rawshdeh, C. T. Cardenas, K. W. Yaseen, A. Maspero and M. A. Omary, Fluorous metal-organic frameworks and nonporous coordination polymers as low- $\mathrm{K}$ dielectrics, Adv. Funct. Mater., 2019, 29, 1904707.

50 F. C. Krebs and H. J. Spanggaard, An exceptional red shift of emission maxima upon fluorine substitution, J. Org. Chem., 2002, 67, 7185.

51 M. A. H. Alamiry, A. C. Benniston, J. Hagon, T. P. L. Winstanley, H. Lemmetyinen and N. V. Tkachenko, The fluorine effect: photophysical properties of borondipyrromethene (bodipy) dyes appended at the meso position with fluorinated aryl groups, RCS Adv., 2012, 2, 4944-4950.

52 S. Galli, A. Maspero, C. Giacobbe, G. Palmisano, L. Nardo, A. Comotti, I. Bassanetti, P. Sozzani and N. Masciocchi, When long bis(pyrazolates) meet late transition metals: structure, stability and adsorption of metal-organic frameworks featuring large parallel channels, J. Mater. Chem. A, 2014, 2, 12208-12221.

53 S. Bracco, F. Castiglioni, A. Comotti, S. Galli, M. Negroni, A. Maspero and P. Sozzani, Ultrafast molecular rotors and their $\mathrm{CO}_{2}$ tuning in MOFs with rod-like ligands, Chem. Eur. J., 2017, 23, 11210-11215.

54 T. H. V. Huynh, M. L. H. Mantel, K. Mikkelsen, A. T. Lindhardt, N. C. Nielsen, D. Otzen and T. A. Skrydstrup, Versatile approach to $\beta$-amyloid fibril-binding compounds exploiting the Shirakawa/Hayashi protocol for trans-alkene synthesis, Org. Lett., 2009, 11, 999-1002.

This journal is $@$ The Royal Society of Chemistry 20xx 
65 S. F. Boys and F. Bernardi, The calculation of small molecular interactions by the differences of separate total energies. Some procedures with reduced errors, Mol. Phys., 1970, 19, 553-566.

66 F. W. Sears, M. W. Zemansky and H. D. Young, University Physics, 6th Ed., Addison-Wesley: 1982.

67 ImageJ, National Institutes of Health, U.S. Department of Health and Human Services, imagej.nih.gov/ij/ (accessed January $10^{\text {th }} 2020$ ).

68 K. Sonogashira, in Metal-Catalyzed Cross-Coupling Reactions Wiley-VCH Verlag GmbH: Weinheim, Germany, 2007; pp. 203229.

55 Q. Lin, D. Meloni, Y. Pan, M. Xia, J. Rodgers, S. Shepard, M. Li, L. Galya, B. Metcalf, T.-Y. Yue, P. Liu and J. Zhou, Enantioselective synthesis of Janus kinase inhibitor INCB018424 via an organocatalytic aza-Michael reaction, Org Lett., 2009, 11, 1999-2002.

56 Bruker (2012). SMART. Bruker AXS Inc., Madison, Wisconsin, USA.

57 Bruker (2012). TWINABS. Bruker AXS Inc., Madison, Wisconsin, USA.

58 L. Krause, R. Herbst-Irmer, G. M. Sheldrick and D. Stalke, Comparison of silver and molybdenum microfocus X-ray sources for single-crystal structure determination, J. Appl. Crystalogr., 2015, 48, 3-10.

59 G. M. Sheldrick, SHELXT - Integrated space-group and crystalstructure determination, Acta Crystallogr. Sect. A, 2015, 71, 38.

60 G. M. Sheldrick, Crystal structure refinement with SHELXL, Acta Crystallogr. Sect. C, 2015, 71, 3-8.

61 O. V. Dolomanov, L. J. Bourhis, R. J. Gildea, J. A. K. Howard and H. Puschmann, OLEX2: A complete structure solution, refinement and analysis program, J. Appl. Crystallogr., 2009, 42, 339-341.

62 C. F. Macrae, I. J. Bruno, J. A. Chisholm, P. R. Edgington, P. McCabe, E. Pidcock, L. Rodriguez-Monge, R. Taylor, J. Van de Streek and P. A. Wood, Mercury CSD 2.0-new features for the visualization and investigation of crystal structures, J. Appl. Crystallogr., 2008, 41, 466-470.

63 A. D. Becke, Density-functional thermochemistry. III. The role of exact exchange, J. Chem. Phys., 1993, 98, 5648-5652.

64 M. J. Frisch, G. W. Trucks, H. B. Schlegel, G. E. Scuseria, M. A. Robb and J. R. Cheeseman, Gaussian09, Revision B.01; Gaussian, Inc.: Pittsburgh, PA, 2003. 